Polarities influence implicit associations between colour and emotion

Claudia Kawai*, Gáspár Lukács ${ }^{\mathrm{a}}$, and Ulrich Ansorgea ${ }^{\mathrm{a}, \mathrm{b}}$

${ }^{a}$ Faculty of Psychology, University of Vienna, Austria; ${ }^{b}$ Vienna Cognitive Science Hub, University of Vienna, Austria

Correspondence concerning this article should be addressed to Claudia Kawai, Faculty of Psychology, University of Vienna, Liebiggasse 5, A-1010 Vienna, Austria. E-mail: claudia.kawai@univie.ac.at 


\section{Polarities Influence Implicit Associations between Colour and Emotion}

Colours are linked to emotional concepts. Research on the effect of red in particular has been extensive, and evidence shows that positive as well as negative associations can be salient in different contexts. In this paper, we investigate the impact of the contextual factor of polarity. According to the polaritycorrespondence principle, negative and positive category poles are assigned to the binary response categories (here positive vs. negative valence) and the perceptual dimension (green vs. red) in a discrimination task. Response facilitation occurs only where the conceptual category (valence) and the perceptual feature (colour) share the same pole (i.e., where both are plus or both are minus). We asked participants $(n=140)$ to classify the valence of green and red words within two types of blocks: (a) where all words were of the same colour (monochromatic conditions) providing no opposition in the perceptual dimension, and (b) where red and green words were randomly mixed (mixed-colour conditions). Our results show that red facilitates responses to negative words when the colour green is present (mixed-colour conditions) but not when it is absent (monochromatic conditions). This is in line with the polarity-correspondence principle, but colourspecific valence-affect associations contribute to the found effects.

Keywords: polarity correspondence; congruence; colour; emotion; valence 


\section{Introduction}

The effect (and affect) of colour has long been-and will likely remain to be-a topic of interest to the public eye, pop culture, and sciences. Accordingly, research has addressed various subject areas, such as colour associations (Clarke \& Costall, 2008; Crozier, 1999; Kaya \& Epps, 2004), affective judgements (Briki \& Hue, 2016; Terwogt \& Hoeksma, 1995), cross-cultural preferences (Adams \& Osgood, 1973; Saito, 1996), and colours' effects on mood (Akers et al., 2012) and behaviour (Labrecque \& Milne, 2012; Lichtenfeld et al., 2012; Wilson, 1966).

Central to the current paper are the dynamic associations linking emotional ${ }^{1}$ valence and colour. Red and green are the colours we will focus on, as research shows that these two colours can vary maximally on the valence (evaluation) dimension (see Schietecat et al., 2018b). Conventionally, financial gains, upward trends, secure situations and agreeing actions are depicted in green colour, and conversely, losses, downward trends, potential danger and disagreeing actions in red colour. Despite this conventional use that connects green to positive contexts and red to its negative counterpart, research on the colour red painted a rather colourful, inconsistent picture.

\subsection{The Red-Effect(s)}

Given the appropriate context, a colour can elicit very different associations, potentially even of opposing valences (e.g., Schloss et al., 2013; see Elliot \& Maier, 2012, for a review of context-dependence of colour). When it comes to explicit associations for red, both negative (anger, danger, blood) and positive (love, passion, warmth) concepts ${ }^{2}$ are salient (Kaya \& Epps, 2004; Jonauskaite et al., 2019; Soriano \& Valenzuela, 2009). Likewise, when it comes to implicit associations, there is evidence for an ambiguous role of red, indicating links to both positive and negative valence. On the one hand, red was found to be related to high social status and prestige (Wu et al., 2018). On the other hand, red was shown to be associated with negativity in general (Kuhbandner \& Pekrun, 2013; Schietecatet al., 2018b), and anger, danger, and failure-related emotion words in particular (Fetterman et al., 2012; Moller et al., 2009; Pravossoudovitch et al., 2014).

Its highly polysemous nature is emphasized by all the different red-effects researchers have reported, some of which suggest a positive, appetitive influence on human behaviour, others an aversive one. Red increases the perceived attractiveness in the opposite sex (Elliot et al., 2010; Elliot \& Niesta, 2008; Pazda et al., 2012; but see 
Francis, 2013; Lehmann \& Calin-Jageman, 2017; Lehmann et al., 2018; Peperkoorn et al., 2016), but also the perceived negativity in affective pictures (Buechner \& Maier, 2016; but see Smajic et al., 2014; Steele, 2014). Even influences on cognitive and physical performance have been reported, in the way that red enhances achievement in detailoriented cognitive tasks (Mehta \& Zhu, 2009), but undermines it in IQ tasks (Elliot et al., 2007; Lichtenfeld et al., 2009) and motor performance (Briki et al., 2015, but see Akers et al., 2012).

Looking at the results, it seems evident that colour works in context, but which exact contextual factors determine if a given colour evokes an appetitive or aversive stimulus evaluation in the beholder is unknown to date. In general, a variety of factors can contribute to the salience or the weight of each particular conceptual dimension of a stimulus to processing (e.g., Ansorge et al., 2014; Elliot \& Maier, 2012; Lebois et al., 2015). One contextual influence responsible for whether or not a conceptual factor becomes salient is task relevance of a stimulus dimension (see Moors \& De Houwer, 2006). Task relevance can be expressed explicitly (e.g., via task instructions), but might sometimes also be of a more subtle nature: to categorise stimuli by alternative responses may highlight features that discriminate between the responses (e.g., their spatial positions) that in turn gate influences of similar stimulus features (e.g., their locations) on performance (cf. Ansorge \& Wühr, 2004). Below, we explicate one such subtle contextual factor related to task relevance that we hypothesise plays a decisive role in colour-valence interactions: polarity correspondence.

\subsection{Polarity Correspondence}

Reviewing the seemingly contradictory results on the colour red, as stated above, one decisive influence of how a colour is perceived, or reacted to, is the presence of opposite pole stimuli. In fact, many studies showed that, in general, congruence or compatibility effects between conceptual and/or perceptual dimensions are present where stimuli from opposite poles on such conceptual and perceptual dimensions are present; however, such congruence effects are absent where only stimuli from one pole of either the conceptual or the perceptual dimension are present (cf. Ansorge \& Wühr, 2004; Wühr et al., 2008). Importantly, studies that investigated the relationship between colour and valence (see above, e.g., Fetterman et al., 2012; Wu et al., 2018), often (albeit not always) did so by opposing one colour (e.g., red) to another chromatic or achromatic colour in the same task (e.g., blue or grey), thereby manipulating colour within blocks and participants. 
These studies typically applied binary-classification tasks, like the implicit association test (IAT; see Greenwald et al., 1998) or other semantic categorisation tasks, requiring bimanual classification responses ('Press key A or key B'). ${ }^{3}$ This is interesting because polarity correspondence as a contextual factor allows congruence effects between conceptual and perceptual dimensions such as emotion, colour, or position that critically depend on the presence of opposing poles in two dimensions (e.g., different valences and different colours) in such binary-classification experiments (Lakens, 2012; Lakens et al., 2012; Proctor \& Cho, 2006).

The rationale behind the polarity correspondence principle is the following: Binary classification of target stimuli that vary on one conceptual dimension by alternative responses (e.g., pressing left key for stimuli above vs. pressing right key for stimuli below fixation) suggests to the participants to code target alternatives as plus (+) poles versus minus (-) poles.

Our first test of the polarity-correspondence principle made use of the dimensions' dichotomy as a necessary precondition for the colour-valence congruence effect: the binary task-relevant as well as an additional binary irrelevant dimension, in our case positive versus negative valence and red versus green colour, respectively, that seems to be required to allow for many compatibility effects in general (cf. Ansorge \& Wühr, 2004) and for a polarity-based congruence effect in particular. In a series of studies, Lakens et al. (2012) showed how an effect of the white-good association only emerged when participants formed a black-bad association in opposition. In line with the necessity of variation on the second dimension involved in this congruence effect, in the absence of black cues, congruence effects for white-good stimuli were no longer observed (Lakens et al., 2012).

While this was a test of brightness-emotion associations, this logic has recently also been applied in an indirect way to the hue-emotion association case of red/green colour-valence congruence effects by Schietecat et al. (2018b). Between tasks, these authors varied if participants had to classify target words by their valence or by their activity value. In a version of the IAT, Schietecat et al. (2018b) found that the change of task reversed the association of the colour red with the respective pole of the task-relevant conceptual dimension: In a binary valence-classification task (positive vs. negative), red was congruent with the - pole (i.e., negative) targets, but in a binary activity-classification task (aggressive vs. calm), red was congruent with the + pole (i.e., active/aggressive). This reversal suggests that the binary classification in one conceptual dimension defined 
by task-relevance is decisive for the direction of the colour-valence association, but the study by Schietecat et al. (2018b) left open if the absence of colour opposition diminishes the colour-valence congruence effects in the predicted way. If polarity correspondence is a decisive factor in the congruence of colour-valence associations, then congruence effect sizes should differ, depending on the presence versus absence of structural overlap between dimensions.

In the current study, the first test of contributions of polarity correspondence to colour-valence congruence effects therefore compared performance in blocks in which we presented red and green colour stimuli intermixed (mixed-colour condition) with the performance in blocks with only a single colour for all stimuli (monochromatic condition), expecting a stronger colour-valence congruence effect in the mixed-colour condition than in the monochromatic condition.

As our second test of the polarity-correspondence principle, we checked our data for the distinct asymmetrical response (time) pattern that is typically predicted in similar bimanual cross-modal classification tasks (Lakens, 2012). The reason of the unequal congruence effect sizes can be explained by the following rationale: to comply with any arbitrary binary classification instructions, participants can assign priority to one of the two available alternatives; that is, they can look for the presence of the prioritized target characteristic (say, for stimuli above as + pole attribute), responding "yes" to the + pole attribute when the search criterion is fulfilled (stimulus is above) and responding "no" to the + pole when the searched-for feature is absent (stimulus is not above); or participants can instead switch to the alternative task of searching for (and responding "yes" to) the less prioritised characteristic (stimulus is below fixation). In either case, for - pole but not for + pole stimuli, switching between responses (see Bertelson, 1965) or between tasks (Rogers \& Monsell, 1995; Wolfe, Butcher, Lee, \& Hyle, 2003) should delay responses. In line with this assumption, where participants consistently assign + poles and - poles to different specific target alternatives, + pole responses (e.g., to stimuli above fixation) are faster than - pole responses (e.g., to stimuli below fixation; see Clark, 1969; Lakens, 2012; Proctor \& Cho, 2006). In the following, we refer to this influence brought about by the polarity-correspondence principle as + pole response facilitation.

Importantly in the context of the present investigation of colour-valence associations, participants apparently assign poles to additional conceptual dimensions of the targets besides their relevant features, as long as these additional conceptual dimensions also vary in a binary way. This is what Proctor and Cho (2006) refer to as 
structural overlap, which occurs when an experimental design allows for a symmetrical mapping across (conceptual/perceptual) dimensions. Returning to the previous example: when participants classify target words appearing above or below a fixation point (variation on the spatial dimension) as positive or negative (variation on the valence dimension), responses are faster to positive targets above fixation than to negative targets above fixation (Meier \& Robinson, 2004). Theoretically, this could be due to contextindependent semantic priming of the task-irrelevant stimulus position by the relevant emotional dimension (i.e., 'good is up' and 'bad is not up/bad is down'). However, in line with the polarity-correspondence principle, these space-valence congruence effects can be missing or even reversed for + pole words below fixation (technically an incongruent condition) compared to - pole words below fixation (technically a congruent condition). As explained by Lakens (2012), + pole response facilitation according to the polaritycorrespondence principle can account for this asymmetric congruence effect (i.e., the presence of the congruence effect for + pole stimuli but its absence or diminution for pole stimuli). First of all, indicative of additional facilitation through the + pole responses on top of the facilitation by polar congruence alone, Lakens found that responses to congruent $+/+$ pole stimuli (here: positive words above fixation) were significantly faster than to congruent -/- pole stimuli (here: negative words below fixation). Secondly, however, in the case of Lakens' (2012) meta-analysis of experiments on space-valence associations, this + pole response facilitation was so strong as to even facilitate responses to incongruent $+/-$ pole stimuli (here: positive words below fixation) relative to congruent -/- pole stimuli (here: negative words below fixation). This abolished the congruence effect under - pole conditions. This asymmetry of the strength of the congruence effect is, thus, also an indication of the polarity-correspondence principle, as it directly follows from + pole response facilitation. To test if polarity correspondence contributes to the colour-valence congruence effect like it does to position-valence congruence, in the current study, we investigated if we find a similar asymmetry of the congruence effect for red and green positive and negative word targets.

\subsection{Congruence Sequence Effect}

For our test of the asymmetry of the colour-valence congruence effect, we also wanted to rule out one alternative explanation: Past research suggested that congruence in the preceding trial $(n-1)$ and emotional valences can jointly influence the congruence effect 
in a current trial $(n)$. In general, congruence effects are stronger following congruent than following incongruent trials (Botvinick et al., 2001; Gratton et al., 1992; Huber-Huber \& Ansorge, 2017; Puccioni \& Vallesi, 2012). These congruence sequence effects are at least partly brought about by cognitive control: an active suppression of the irrelevant dimension and more attention to the relevant dimension following an incongruent/conflicting trial than following a congruent trial without conflict (e.g., Huber-Huber \& Ansorge, 2017). Importantly, this kind of cognitive control critically depends on the negative affect elicited by the conflict (Hobson et al., 2014; Inzlicht et al., 2015; Schouppe et al., 2015; van Steenbergen et al., 2009, 2010). First of all, conflict elicits negative affect as indicated by priming of negative judgments through conflicting stimuli (cf. Dreisbach \& Fischer, 2012; Fritz \& Dreisbach, 2013; Goller et al., 2019). Secondly, modifying the affective response to the conflicting stimulus changes the amount of exerted cognitive control (e.g., de Bruijn et al., 2004; Hobson et al., 2014; van Steenbergen et al., 2009, 2010). For example, administration of an anxiolytic drug diminishes the error-related negativity (ERN) that follows an erroneous response in a conflict task (de Bruijn et al., 2004). Likewise, van Steenbergen et al. (2009) found reduced congruence sequence effects if participants received a monetary gain. The upshot of such investigations is that the valence of the target stimulus presented following a conflicting trial could theoretically influence the size of conflict regulation and, thus, the average size of the congruence effect, too. Following a preceding incongruent trial $n-1$, presenting a negative target word in a current trial $n$ could semantically prime conflictelicited negative affect and, hence, conflict regulation. In contrast, presenting a positive target word in a current trial $n$ would not semantically prime and possibly even counteract conflict-elicited negative affect and, hence, diminish cognitive control. As a consequence, the asymmetry of the congruence effect predicted by the polaritycorrespondence principle - stronger congruence effects with positive than with negative words - could also be due to more cognitive control and resulting smaller average congruence effects with negative than positive target words. This would be reflected in an interaction between (1) valence, (2) congruence in the preceding trial, and (3) congruence in the current trial: lower congruence effects following incongruent trials, more so with negative than with positive words. Importantly, such an influence goes hand in hand with the predicted two-way interaction between word valence and congruence (in the current trial): a diminution of the average congruence effect for negative words compared to positive words, but for different reasons than assumed by the polarity- 
correspondence principle.

In an explorative analysis, this potential alternative explanation of the congruence-effect asymmetry was therefore tested, too. To that end, we analysed congruence effects as a function of congruence in a preceding trial (congruence in $n-1$ ). Only when this variable is included in our analysis can we eventually decide if the polarity-correspondence principle or the valence-dependency of conflict regulation that we sketched above accounts better for the expected asymmetry of the congruence effect. Our criterion for this test will be the presence versus absence of the significant three-way interaction between target valence, congruence in trial $n-1$, and congruence in trial $n$. This interaction should be significant if congruence between the affective state and preceding conflict account for the asymmetry of the congruence effect. In contrast, the three-way interaction should be absent when the polarity-correspondence principle accounts for the expected asymmetry of the congruence effect. According to our knowledge, no polarity-correspondence study has tested for this potential alternative explanation to date. This is why we found it necessary to analyse congruence sequence effects as a function of valence.

\section{Methods}

\subsection{Participants}

Based on effect sizes in a previous pilot similar to the current study (Lohmann \& Jorschick, 2015) of Cohen's $f=.86$, for a power of $.95, \alpha=.05$, the suggested minimum sample size (using the G*Power tool; Faul et al., 2009) is 20 participants (for the mixed colour condition). Taking into consideration that in the monochromatic blocks, Colour was a between-participants, not a within-participant variable, we estimated that 40-60 participants per condition would be sufficient to gauge whether any differences are present in the conditions. Keeping the participant number comparable across the three conditions, we aimed for a total of 120-180 participants. $^{4}$

In total, 145 students at University of Vienna (27 men, $M_{\text {age }}=20.86$ years, $S D_{\text {age }}$ \pm 2.85 ) participated in exchange for course credit. Participants were randomly assigned to one of the block order conditions (see Table 1). 
Table 1. Block Order and Number of Participants Assigned to Each Experimental Condition.

\begin{tabular}{lllll}
\hline Condition & $1^{\text {st }}$ Block & $2^{\text {nd }}$ Block & $3^{\text {rd }}$ Block & $N$ \\
\hline 1 & Mono red & Mono green & Mixed & 50 \\
2 & Mono green & Mono red & Mixed & 47 \\
3 & Mixed & Mono red & Mono green & 23 \\
4 & Mixed & Mono green & Mono red & 24 \\
Total & & & & $\mathbf{1 4 5}$ \\
\hline
\end{tabular}

Note. Our main interest lay in effects in the first experimental block, in which case Conditions 3 and 4 were identical (both starting with mixed blocks). We therefore collected for Conditions 3 and 4 in sum approximately the same number of participants as we did for Condition 1 and Condition 2 respectively.

\subsection{Materials}

We selected 60 positive and 60 negative German words from the Berlin Affective Word List Reloaded (BAWL-R; Võ et al., 2009). We classified words as positive when the mean emotion value was $\geq 0.6$ and as negative when the mean emotion value was $\leq-0.6$. The number of nouns, verbs and adjectives was balanced, and values for word arousal, imageability, letter count and word frequency (Brysbaert et al., 2011) were kept constant across the two valence categories (see Table 2). A list with all stimuli used is provided in the Appendix. To ensure equiluminance of stimulus colour, brightness values for red, green, and grey (background colour) were measured with a spectrophotometer (X-Rite i1XTreme, Grand Rapids, MI, USA) for each of the five monitors used and colour values were selected accordingly (see Table 3 for the $\mathrm{L}^{*} \mathrm{C}^{*} \mathrm{~h}^{*}$ values, as referenced in the CIE LCh colour space; a detailed list with the values per monitor is provided in the materials section on osf.io/c4zry). Instruction colour, which was used for all text not constituting experimental stimuli, was in a light blue hue, and was, depending on the luminancecontrolled colours picked for each monitor, calculated by averaging the reds and greens and inverting the result for better readability against the grey background. The size of the coloured word stimuli was set to 50 pixels (angular size $1.45^{\circ}$ ), with a fixed viewing distance between eye and centre display of $60 \mathrm{~cm}$, assured via the utilization of chin rests. 
Table 2. Number of Positive and Negative Stimuli per Word Category and Mean Values for Valence, Arousal, Imageability and Letters Taken from the Berlin Affective Word List Reloaded (BAWL-R) Database, and Log Word Frequencies (Subtlex Lg) Taken from the SUBTLEX Database.

\begin{tabular}{lllllllll}
\hline & $\begin{array}{l}\text { Emotion } \\
\text { Value }\end{array}$ & $\begin{array}{l}\text { Arousal } \\
\text { Value }\end{array}$ & $\begin{array}{l}\text { Image } \\
\text { Value }\end{array}$ & Letters & Frequency & Nouns & Verbs & Adjectives \\
& 1.67 & 2.98 & 3.94 & 6.83 & 2.37 & 20 & 20 & 20 \\
\hline Positive & 1.67 & 3.98 & 6.77 & 2.29 & 20 & 20 & 20 \\
Negative & -1.53 & 3.12 & 3.98 & & & \\
\hline
\end{tabular}

Table 3. Average Values for Lightness ( $\left.\mathrm{L}^{*}\right)$, Chroma $\left(\mathrm{C}^{*}\right)$, and Hue ( $\mathrm{h}^{*}$ Specified in Degrees) (according to the CIE LCh Colour Model) of the Red, Green, and Grey Colour Used in the Experiment. Saturation (sat) is Computed as $\mathrm{C}^{*} / \mathrm{L}^{*}$ (see Wilms \& Oberfeld, 2018).

\begin{tabular}{lllll}
\hline & $\mathrm{L}^{*}$ & $\mathrm{C}^{*}$ & $\mathrm{~h}^{*}$ & sat \\
\hline Red & 51.17 & 102 & 40.14 & 1.99 \\
Green & 51.81 & 74.03 & 134.39 & 1.43 \\
Grey & 35.37 & 0 & 145.10 & 0 \\
\hline
\end{tabular}

\subsection{Apparatus}

During the study, stimuli were presented on five 19-inch LCD monitors with a 1,280 $\times$ 1,024 resolution and a refresh rate of $60 \mathrm{~Hz}$. Responses and associated response times (RTs) were registered via serial QWERTZ computer keyboards.

\subsection{Procedure}

Testing was conducted in a dimly lit laboratory room. Upon arrival, participants were welcomed and placed at one of five identical PCs, running PsychoPy2 software (Peirce, 2007). After signing the consent forms, participants were instructed to place their chin on the chin rest and start the experiment. Instructions were presented on screen. The study consisted of two tasks: an initial valence-rating task and a subsequent binary valenceclassification task. The experimental session ended with a short test for colour deficiency. 


\subsubsection{Valence-Rating Task}

To ensure that the valence provided in the BAWL-R database matched participants' perceived valence, a rating task ('Please rate the valence of the word.') was employed before the binary classification task. Participants judged each of 120 potential target words once. Per each trial, one word was presented in the instruction colour, in capital letters, and in randomised order, centred above a 10-point rating scale with the endpoints being labelled very negative ('sehr negativ') on the left side of the screen and very positive ('sehr positiv') on the right side. Participants had to move the mouse cursor to the tick mark corresponding with their rating and confirm the selected valence value with a leftclick. Words that received a rating below six were classified as 'rated negative' and appeared in a text box on the left side of the screen, below the negative scale label; words with a rating of six or higher were coded as 'rated positive' and appeared in a text box on the right side of the screen, below the positive scale label. Each word was presented for a variable duration (until the judgment was made). There were no time constraints for this task and participants were informed thusly. After participants' rating of all words, the 50 most positively and the 50 most negatively rated words were selected for the upcoming valence-categorisation task. If the number of words per category was lower than 50 , the shortest word list (list of words rated positive or list of words rated negative by the participant) determined the overall number of stimuli that appeared in the subsequent task. For an equal number of positive and negative items, the word list of the remaining valence category was trimmed accordingly. For example, if a participant rated 43 of the 60 positive words (per BAWL-R database) as positive and 52 of the 60 negative words as negative, then the 43 positive-rated words were selected for the participant's valencecategorisation task, as well as the same number of negative-rated words, in which case the total number of items would be $43+43=86$ items. ${ }^{5}$ The maximum of stimuli presented in the categorisation task was 100 , while there was no minimum of items specified in the experiment. For data analysis, however, we set a lower boundary at a minimum of 40 words as participant exclusion criterion (see Data Collection and Analysis).

\subsubsection{Binary Valence-Classification Task}

The binary classification task started after participants had read the instructions on the monitor, which informed them that, per each trial, they would be presented with a single 
word (which they had previously seen in the valence-rating task) at screen centre. Each target was shown for a maximum of $2 \mathrm{~s}$. For each word, participants judged the valence (Is this word positive or negative?) and indicated their choice by pressing either the ' $\mathrm{E}$ ' (for positive valence) or the 'I' (for negative valence) key on a standard QWERTZ keyboard. Key assignment was balanced across participants. We did not mention to participants beforehand that words would be presented in different colours. In addition to an initial 10-trial practice, the binary classification task comprised three experimental blocks in total: one block with each word (out of the max. 100) presented once in only red colour ('mono red'), one block with each word presented once in green ('mono green'), and a mixed-colour block with each word presented twice, once in red and once in green font colour. Depending on the participants' assigned condition, order of blocks varied (see Table 1). Stimuli were presented in randomised order, with the restrictions that not more than five words of the same valence (in all blocks) and colour (in the mixed blocks) were presented in a row. Participants completed the entire experiment in less than $30 \mathrm{~min}$.

\subsubsection{Test for Colour Deficiency}

After participants completed the valence rating and all three blocks of the binary valenceclassification task, they were asked to enter the numbers printed on three colour plates, which were displayed on the computer screen (pictures are provided as supplementary material under osf.io/c4zry). These pictures were computer-screen versions of the Ishihara colour plates. Note that this is a non-standardized assessment of the Ishihara test for colour deficiency and may not give accurate results as for the presence or absence of colour-blindness. However, since our experimental setup also made use of computerdisplay colours, we opted to include this control measure, in addition to self-reported colour deficiencies. Upon entering all three numbers, participants were debriefed in written form and the experiment ended.

\subsection{Data Collection and Analysis}

Data from the previous rating task was not analysed further, since it only served to ensure the participants' valence judgment. Data from all three blocks of the valenceclassification task was analysed, but analyses reported below were restricted to the first experimental blocks from the valence-categorisation task because by the time of 
commencing mixed-colour blocks, colour polarity was established and blocks in the monochromatic condition were no longer free from potential transfer confounds. However, we provide analyses of the data from all three experimental blocks on the project's OSF page osf.io/c4zry. We also ran a more conservative analysis of the mixed colour blocks, where colour switch trials (a red word following a green word, or vice versa) were excluded from the data. We did this to ensure that congruence effects do not only result from colour switch trials but are also present in colour-repetition trials.

Criteria for data exclusion were as follows: Data from participants who did not reach the full score in our colour-deficiency test or were self-reportedly colour-blind were discarded (four participants). Furthermore, if participants, during the rating task, classified less than 40 items in each valence category in accordance with the valence given in the BAWL-R database, their data was not analysed further (one participant). The exclusion criterion for participants' accuracy was $75 \%$, but since no participant had an overall accuracy rate lower than this threshold, no further participants had to be excluded, leaving a total of $n=140$ participants. Mean error rate (ER) over all blocks of the categorisation task was $6.0 \%$. Trials with RTs below $150 \mathrm{~ms}$ and above $2 \mathrm{~s}$ were excluded from analysis $(<1 \%)$. In the congruence sequence effect analyses, trials with previously erroneous or too slow answers were excluded (e.g., if the response to trial $n-1$ was incorrect and thus excluded from analysis, trial $n$ was excluded as well). For RT analyses, correct median RTs were taken, as the median is less sensitive to disproportionately slow responses than the mean. For error analyses, arcsine square root transformed error rates were used, as this stabilises and normalises variances of proportional data (Sokal \& Rohlf, 1981). Additionally, in Table 4 and 5, we report the untransformed mean ERs for an easier understanding of means over conditions.

\subsubsection{Effect Sizes and Statistical Significance}

To demonstrate the magnitude of the observed effects, partial eta-squared $\left(\eta_{\mathrm{p}}^{2}\right)$ values, $90 \%$ confidence intervals $(\mathrm{CI})$, and generalised eta-squared $\left(\eta_{\mathrm{G}}^{2}\right)$ are reported for $F$-tests (Steiger, 2004). We report Bayes factors (as $\mathrm{BF}_{10}$ when supporting difference, and $\mathrm{BF}_{01}$ when supporting equivalence) using the default $r$-scale of .707 (Morey $\&$ Rouder, 2018). In case of analyses of variance (ANOVAs), we report inclusion BFs based on matched models (Makowski et al., 2019; Mathôt, 2017). Where applicable, we report Welchcorrected $t$-tests (Delacre et al., 2017) with corresponding Cohen's $d$ values (Lakens, 
2013). We used the conventional alpha level of .05 for all statistical significance tests. All analyses were conducted in R (R Core Team, 2019; via: Kelley, 2019; Lawrence, 2016; Lukács, 2019; Morey \& Rouder, 2018)

\section{Results}

Five participants were excluded because they violated the inclusion criteria for data analysis as stated above. Key assignment influenced neither RTs nor ERs (all $F<1$ ) and is not discussed further.

We conducted the preregistered analyses with minor, necessary modifications explicitly specified for the respective tests. The preregistration and all supplementary materials, together with the statistical analyses of all-block data and a more conservative analysis of the mixed-colour blocks including only colour-repetition trials (no colour switch trials), are available under the project's Open Science Framework (Foster \& Deardorff, 2017) page osf.io/c4zry. By and large, the more conservative analysis of only the colour repetition trials in the mixed-colour condition as well as the all-block analysis confirmed the results of the present analyses.

\subsection{Monochromatic Condition}

If polarity on two dimensions is required for congruence effects to emerge, we would expect no congruence effect in the red and green monochromatic conditions, where colour does not vary across trials. In contrast, if polarity correspondence has no effect on the weight of the colours as modifiers of valence processing, then a more typical colourvalence congruence effect, with green-positive and maybe weaker red-negative associations, should show in the monochromatic data. Means of median correct RTs and ERs were calculated per valence, colour, and block condition and are given in Table 4. Figures 1 and 2 illustrate the RTs and ERs, respectively, with results for the monochromatic blocks printed on the left side, and results for the mixed-colour blocks printed on the right.

\subsubsection{Reaction Times (RTs)}

Our analysis was restricted to the participants that started with either the red or the green monochromatic block (see Table 1, Conditions 1 and 2) because the performance of these participants was free from any potential carry-over of alternative polar assignments of the 
colours from a preceding mixed colour-block. We conducted a two (between-participants Colour: red vs. green) $\times$ two (within-participant Valence: positive vs. negative) mixeddesign ANOVA on the correct binary classification times (RTs) of the monochromatic (mono red, mono green) blocks. Colour did not influence RTs significantly, $F(1,93)=$ $2.29, p=.134, \eta_{\mathrm{p}}^{2}=.024,90 \% \mathrm{CI}[0, .095], \eta_{\mathrm{G}}^{2}=.022, \mathrm{BF}_{10}=1.40$. There was a significant main effect for Valence, $F(1,93)=22.79, p<.001, \eta_{\mathrm{p}}^{2}=.197,90 \% \mathrm{CI}[.088, .308]$, $\eta_{\mathrm{G}}^{2}=$ $.016, \mathrm{BF}_{10}=2766.49$, with positive words being responded to faster $\left(M_{\mathrm{RT}}=706 \mathrm{~ms}\right)$ than negative words $\left(M_{\mathrm{RT}}=729 \mathrm{~ms}\right)$, a finding in line with + pole response facilitation (Lakens, 2012). ${ }^{6}$ The interaction between Valence and Colour was not significant, $F(1$, $93)=1.41, p=.237, \eta_{\mathrm{p}}^{2}=.015,90 \% \mathrm{CI}[0, .078], \eta_{\mathrm{G}}^{2}=.001, \mathrm{BF}_{01}=2.98$. This is in line with the predicted absence of polarity-correspondence effects where only one of the involved dimensions (i.e., valence) varied but the other (i.e., colour) did not.

RTs to red positive words were slower than RTs to green positive words, but RTs to red targets still showed the typical + pole response facilitation for positive red relative to negative red words. Thus, we do not find any evidence for an automatic red-negativity link. This could be due to a lack of any colour-valence congruence effect in the absence of opposing green colours in the monochromatic red blocks. However, this could have also reflected a general red-positivity association of maybe somewhat smaller size than in the case of green stimuli. To investigate, if there could be red-positivity and greenpositivity links, we ran two paired $t$-tests, one for red-monochromatic and one for the green-monochromatic conditions, where we compared the RTs for negative versus positive words.

Despite our decent sample size, in the red-monochromatic condition, there was only weak evidence for a difference in RTs, $t(49)=2.41, p=.020, d_{\text {within }}=0.34,95 \% \mathrm{CI}$ $[0.05,0.62], \mathrm{BF}_{10}=2.11$. In the green-monochromatic condition, evidence for a difference in RTs was strong, $t(44)=4.72, p<.001, d_{\text {within }}=0.70,95 \%$ CI $[0.37,1.03]$, $\mathrm{BF}_{10}=861.62$.

In light of the relatively weak evidence in the red-monochromatic condition, we cannot confidently corroborate the hypothesis that red is associated with positive valence. This lack of an automatic red-positivity or an automatic red-negativity link in a nonpolarity context could be an indication for an ambiguous role of the colour red when it comes to valence associations, when it is perceived in isolation instead of in opposition 
to another colour. For an illustration of the RT data from the monochromatic conditions see Figure 1, left panel.

\subsubsection{Error Rates (ERs)}

We computed an ANOVA with the same variables on the arcsine transformed ERs. Results corroborated the RT findings. Colour did not influence ERs significantly, $F(1$, $93)=0.64, p=.426, \eta_{\mathrm{p}}^{2}=.007,90 \% \mathrm{CI}[0, .059], \eta_{\mathrm{G}}^{2}=.005, \mathrm{BF}_{01}=3.31$. There was a significant main effect for Valence, $F(1,93)=21.01, p<.001, \eta_{\mathrm{p}}^{2}=.184,90 \%$ CI [.078, $.295], \eta_{\mathrm{G}}^{2}=.066, \mathrm{BF}_{10}=1238.00$, with less accurate responses to negative $\left(M_{\mathrm{ER}}=7.4 \%\right)$ than to positive stimuli $\left(M_{\mathrm{ER}}=4.9 \%\right)$. The interaction between Colour and Valence was not significant, $F(1,93)=2.37, p=.127, \eta_{\mathrm{p}}^{2}=.025,90 \% \mathrm{CI}[0, .097], \eta_{\mathrm{G}}^{2}=.008, \mathrm{BF}_{01}=$ 1.69. For an illustration of the ER data from the monochromatic conditions see Figure 2, left panel.

\subsection{Mixed-Colour Condition}

Above, we have shown that the Colour $\times$ Valence interaction is not significant in case of monochromatic blocks. Now we tested the same interaction in case of mixed-colour blocks (again, only for the conditions where the mixed-colour block was the first experimental block, i.e., Conditions 3 and 4). Since both colours are present during the mixed-colour block, colour and valence were within-participant variables. Contrary to the monochromatic blocks, in the mixed-colour condition, colour polarity is present, since stimuli are presented intermixed in either red or green. In such a context, the polarity correspondence principle (Lakens, 2012) predicts the prevalence of congruence effects (i.e., better performance in congruent than incongruent conditions), in the way that through polar assignments, green should share the + pole with positive valence and red should share the - pole with negative valence. Such a congruence effect should then be reflected in the form of a significant interaction between Colour and Valence. ${ }^{7}$

\subsubsection{Reaction Times (RTs)}

The results showed a main effect for Valence, $F(1,44)=30.71, p<.001, \eta_{\mathrm{p}}^{2}=.411,90 \%$ CI $[0.220,0.545], \eta_{\mathrm{G}}^{2}=.024, \mathrm{BF}_{10}=1601.38$, and a weaker main effect for Colour, $F(1$, $44)=5.08, p=.029, \eta_{p}^{2}=.103,90 \%$ CI $[.006, .252], \eta_{G}^{2}=.003, \mathrm{BF}_{01}=2.30$. In line with 
the expectations based on the influence of the polarity-correspondence principle, the Colour $\times$ Valence interaction was significant, $F(1,44)=61.81, p<.001, \eta_{\mathrm{p}}^{2}=.584,90 \%$ CI $[.413, .684], \eta_{\mathrm{G}}^{2}=.053, \mathrm{BF}_{10}=4.26 \times 10^{11}$. Two paired $t$-tests showed the predicted congruence effect in line with previous research: In green colour, positive words were classified faster than negative words, $M_{\mathrm{RT}} \pm S D=690.91 \pm 78.43$ vs. $754.81 \pm 81.69$, respectively, with $t(44)=10.39, p<.001, d_{\text {within }}=1.55,95 \% \mathrm{CI}[1.11,1.98], \mathrm{BF}_{10}=3.88$ $\times 10^{10}$, while in red colour, negative words were classified faster than positive words, $M_{\mathrm{RT}} \pm S D=724.58 \pm 87.31$ vs. $737.62 \pm 82.46$, respectively, with $t(44)=-1.81, p=.078$, $d_{\text {within }}=-0.27,95 \% \mathrm{CI}[-0.57,0.03], \mathrm{BF}_{01}=1.39$. Although the latter post-hoc test failed to reach significance, the trend was in the expected direction, in line with our hypothesis of red-negative stimuli being responded to faster than red-positive stimuli, when opposed to green colour. Together, these stronger congruence effects for the green than for the red target words in the mixed-colour condition confirmed the presence of a congruence-effect asymmetry with stronger effects for the + pole than for the - pole stimuli.

The results for the RT data from the mixed-colour conditions are illustrated in Figure 1, right panel.

Table 4. Means (SD in Parentheses) of the Median Reaction Times (MRT; in ms) and Mean Errors (MER; in \%) of the First Experimental Block per Colour and Valence Conditions.

Monochromatic

Mixed Colour

\begin{tabular}{llllll}
\cline { 3 - 5 } Colour & Valence & $M_{\mathrm{RT}}(S D)$ & $M_{\mathrm{ER}}(S D)$ & $M_{\mathrm{RT}}(S D)$ & $M_{\mathrm{ER}}(S D)$ \\
\hline Green & & & & \\
& Positive & $689(86)$ & $4.8(4.2)$ & $691(78)$ & $3.0(4.1)$ \\
& Negative & $718(85)$ & $8.1(5.3)$ & $755(82)$ & $9.8(7.8)$ \\
\multirow{2}{*}{ Red } & & & & \\
& & & & $738(82)$ & $8.1(5.8)$ \\
& Positive & $722(93)$ & $4.9(3.9)$ & $725(87)$ & $3.1(3.0)$ \\
\hline
\end{tabular}




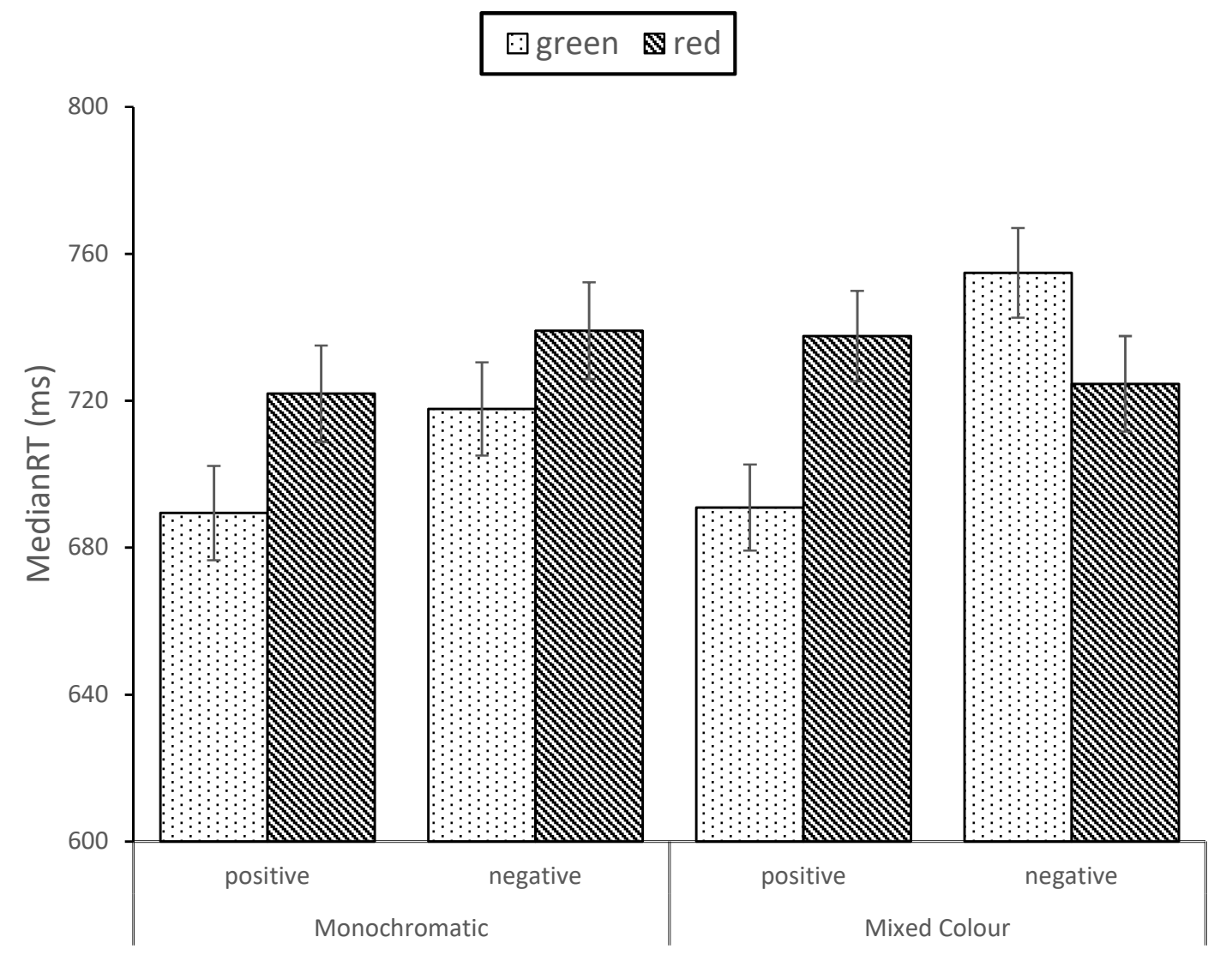

Figure 1. Median reaction times (RTs) for positive and negative words presented in red and green font colour in the mixed-colour versus monochromatic conditions. Error bars show the $S E$ of the means.

\subsubsection{Error Rates (ERs)}

We conducted an ANOVA with the same variables on the arcsine transformed ERs. Neither Valence nor Colour elicited a significant main effect, $F(1,44)=1.22, p=.275$, $\eta_{\mathrm{p}}^{2}=.027,90 \% \mathrm{CI}[0, .142], \eta_{\mathrm{G}}^{2}=.005, \mathrm{BF}_{01}=4.33, F(1,44)=0.02, p=.882, \eta_{\mathrm{p}}^{2}<.001$, $90 \%$ CI $[0, .036], \eta_{\mathrm{G}}^{2}<.001, \mathrm{BF}_{01}=6.23$, respectively. The interaction between Colour and Valence was significant, $F(1,44)=78.39, p<.001, \eta_{\mathrm{p}}^{2}=.640,90 \%$ CI [.483, .727], $\eta_{\mathrm{G}}^{2}=.248, \mathrm{BF}_{10}=1.04 \times 10^{14}$. We performed two paired $t$-tests on the arcsine transformed ERs. They corroborated the expected congruence effect: In green colour, positive words were classified more accurately than negative words, $M_{\mathrm{ER}} \operatorname{asin} \pm S D=0.13 \pm 0.12$ vs. $0.29 \pm 0.15$, respectively, with $t(44)=6.65, p<.001, d_{\text {within }}=0.99,95 \%$ CI $[0.63,1.34]$, $\mathrm{BF}_{10}=3.62 \times 10^{5}$, while in red colour, negative words were classified more accurately than positive words, $M_{\mathrm{ER}}$ asin $\pm S D=0.15 \pm 0.10$ vs. $0.27 \pm 0.11$, respectively, with $t(44)=-$ 
$5.94, p<.001, d_{\text {within }}=-0.89,95 \%$ CI $[-1.23,-0.54], \mathrm{BF}_{10}=3.84 \times 10^{4}$. Unlike the RTs, error rates showed a quite symmetrical cross-interaction between Colour and Valence, and consequently, the size of the congruence effects. The results for the error data from the mixed-colour conditions are illustrated in Figure 2, right panel.

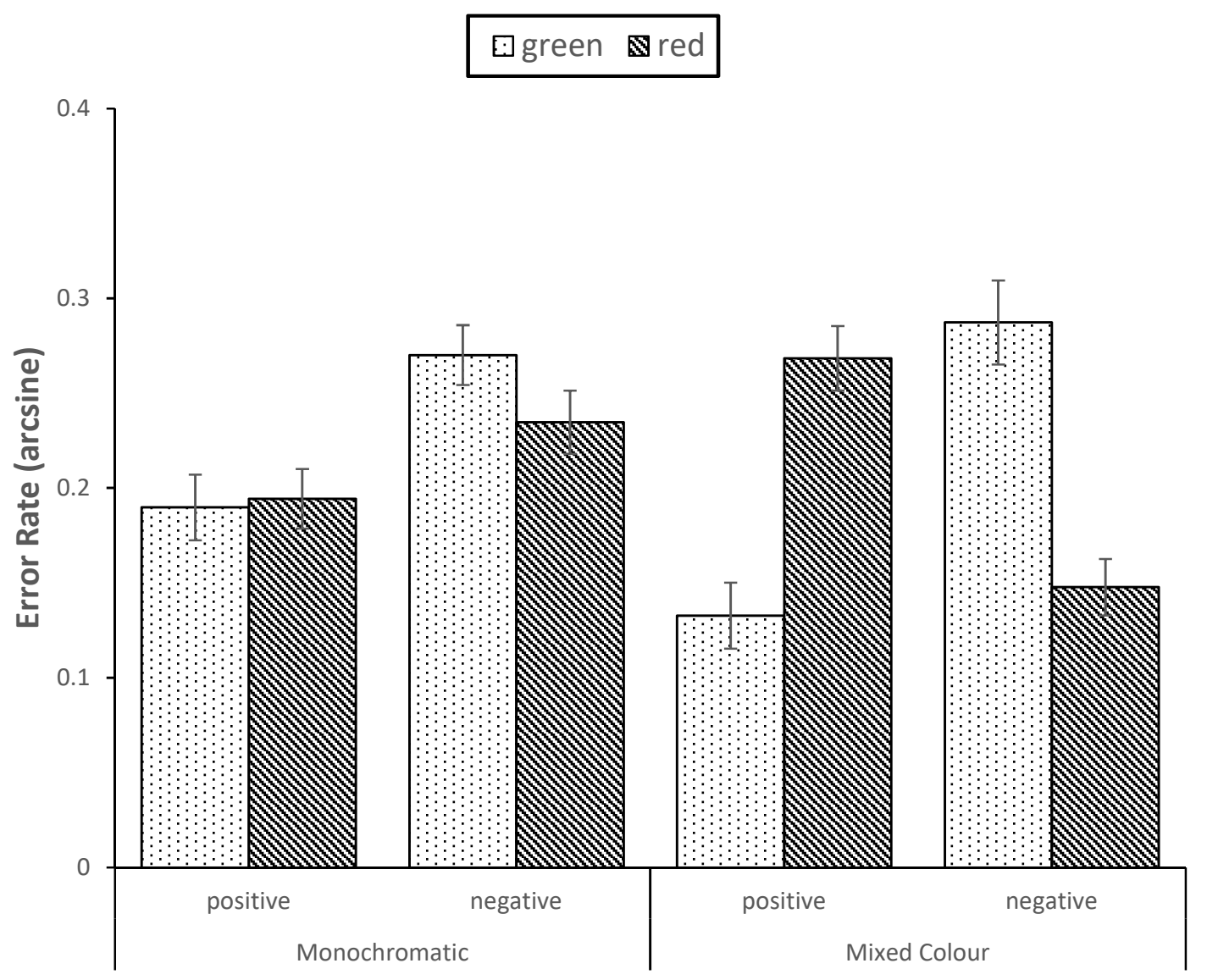

Figure 2. Arcsine transformed error rates for positive and negative words presented in red and green font colour in the mixed-colour versus monochromatic conditions. Error bars show the $S E$ of the means.

\subsection{Congruence Effect Sizes}

To quantify the impact of polarity on the congruence effect, we ran two separate analyses, one for red stimuli and one for green stimuli; this means, we restricted the data taken from the mixed-colour blocks to only one of the colours in turn and contrasted it with the data from the monochromatic condition of the corresponding colour (green-monochromatic versus green-mixed trials; red-monochromatic vs. red-mixed trials). This was done, as the separate ANOVAs for monochromatic blocks and mixed-colour blocks reported above 
differed from each other by how colour was operationalised - as a between-participants variable (in the monochromatic blocks) or as a within-participant variable (in the mixedcolour blocks) - and as this might have affected the power or sensitivity of the designs differently in the two blocks (with arguably more sensitivity for any interactions in the mixed-colour than in the monochromatic blocks). Following the results from Analysis 3.2, for each colour, we coded the trials for Congruence in the way that for green, positive trials were congruent and negative trials were incongruent; conversely, for red, negative trials were congruent trials and positive trials were incongruent.

If polarity, which is only present in the mixed-colour but not in the monochromatic condition, affects responses to stimuli, we would again expect trials from the mixed-colour condition to show a larger congruence effect (difference between incongruent and congruent trials) than from the monochromatic condition, respectively, for each of the two colours.

\subsubsection{Red-monochromatic versus Red-mixed Trials}

Reaction times (RTS). A two (within-participant Congruence: congruent vs. incongruent) $\times$ two (between-participants Condition: monochromatic-red vs. mixed-red) mixed variables ANOVA was conducted on the correct RTs from all red-coloured target trials from the first experimental block, comparing Condition 1 (monochromatic red) to Conditions 3 and 4 (mixed-coloured). The results showed no main effect for Congruence, $F(1,93)=0.31, p=.576, \eta_{\mathrm{p}}^{2}=.003,90 \% \mathrm{CI}[0, .047], \eta_{\mathrm{G}}^{2}<.001, \mathrm{BF}_{01}=5.63$, and no main effect for Condition, $F(1,93)=0.00, p=.972, \eta_{\mathrm{p}}^{2}<.001,90 \% \mathrm{CI}[0,<.001], \eta_{\mathrm{G}}^{2}<$ $.001, \mathrm{BF}_{01}=2.43$. Importantly, despite partly resting on a between-participants variable, the Congruence $\times$ Condition interaction was significant, $F(1,93)=8.83, p=.004, \eta_{\mathrm{p}}^{2}=$ $.087,90 \% \mathrm{CI}[.017, .185], \eta_{\mathrm{G}}^{2}=.007, \mathrm{BF}_{10}=9.79$. As for congruence effects' sizes: Incongruent minus congruent RTs showed an effect of $13 \mathrm{~ms}$ for red in the mixed-colour condition, demonstrating shorter RTs to congruent (negative) than incongruent (positive) stimuli. In contrast, an "inverted congruence effect" of $-17 \mathrm{~ms}$, with faster responses to incongruent than to congruent trials, was found in the red monochromatic condition. The negative effect reflected the typical + pole response facilitation of the valence targets in the absence of the antagonistic colour pole (here: green), showing faster responses to positive (here incongruent) than negative (here congruent) words. Alternatively, as mentioned above, the responses to red targets in the monochromatic blocks might have 
reflected a red-positivity association that can only be unveiled once the context of an antagonistic colour pole is missing.

Error rates $(E R s)$. ERs showed no significant main effect for Condition, $F(1,93)=0.13$, $p=.723, \eta_{\mathrm{p}}^{2}=.001,90 \% \mathrm{CI}[0, .036], \eta_{\mathrm{G}}^{2}=.001, \mathrm{BF}_{01}=4.59$, but a significant main effect for Congruence, $F(1,93)=6.80, p=.011, \eta_{\mathrm{p}}^{2}=.068,90 \%$ CI $[.009, .161], \eta_{\mathrm{G}}^{2}=.026$, $\mathrm{BF}_{10}=1.99$. Importantly, the interaction between Congruence and Condition was significant, $F(1,93)=34.14, p<.001, \eta_{\mathrm{p}}^{2}=.269,90 \% \mathrm{CI}[.147, .379], \eta_{\mathrm{G}}^{2}=.118, \mathrm{BF}_{10}$ $=3.36 \times 10^{5}$, demonstrating also in the error data a larger congruence effect in the red trials from the mixed-colour condition, with a difference of $5.0 \%$, than in the red trials from the monochromatic condition, with a difference of $-1.7 \%$.

\subsubsection{Green-monochromatic versus Green-mixed Trials}

Reaction times (RTS). A two (within-participant Congruence: congruent vs. incongruent) $\times$ two (between-participants Condition: monochromatic-green vs. mixed-colour green) mixed variables ANOVA was conducted on the RTs from all green-coloured trials from the first experimental block comparing Condition 2 (monochromatic green) to Conditions 3 and 4 (mixed-coloured). The main effect for Condition was not significant, $F(1,88)=$ $1.29, p=.258, \eta_{\mathrm{p}}^{2}=.014,90 \%$ CI $[0, .079], \eta_{\mathrm{G}}^{2}=.014, \mathrm{BF}_{01}=1.51$. The main effect for Congruence was significant, $F(1,88)=115.14, p<.001, \eta_{\mathrm{p}}^{2}=.567,90 \%$ CI [.451, .646], $\eta_{\mathrm{G}}^{2}=.073, \mathrm{BF}_{10}=6.53 \times 10^{12}$. Importantly, the interaction between Congruence and Condition was also significant, $F(1,88)=17.12, p<.001, \eta_{\mathrm{p}}^{2}=.163,90 \%$ CI [.060, .275], $\eta_{\mathrm{G}}^{2}=.012, \mathrm{BF}_{10}=222.53 . \mathrm{RT}$ difference comparison (mean of incongruent minus mean of congruent trials) showed that the size of the congruence effect in the mixed-colour condition was $64 \mathrm{~ms}$, while in the green monochromatic condition it was only $29 \mathrm{~ms}$. This latter RT difference was probably no congruence effect at all, as it could have reflected the typical + pole response facilitation of the positive stimuli in general. However, the residual facilitation of RTs to green positive relative to green negative words that we found in the monochromatic blocks could have likewise reflected the more consistent coding of the colour green as positive, regardless of whether or not the context included an antagonistic colour pole (here: red). 
Error rates (ERs). ERs showed a similar pattern. The main effect for Condition was not significant, $F(1,88)=0.87, p=.355, \eta_{\mathrm{p}}^{2}=.010,90 \% \mathrm{CI}[0, .069], \eta_{\mathrm{G}}^{2}=.007, \mathrm{BF}_{01}=3.01$. The main effect for Congruence was significant, $F(1,88)=64.49, p<.001, \eta_{\mathrm{p}}^{2}=.423$, $90 \%$ CI $[.292, .523], \eta_{\mathrm{G}}^{2}=.189, \mathrm{BF}_{10}=2.07 \times 10^{9}$, showing more accurate responses to congruent stimuli (i.e., positive stimuli in green). Importantly, the interaction between Congruence and Condition was significant, $F(1,88)=6.44, p=.013, \eta_{\mathrm{p}}^{2}=.068,90 \% \mathrm{CI}$ $[.008, .164], \eta_{\mathrm{G}}^{2}=.023, \mathrm{BF}_{10}=3.50$. Mean ERs corroborated the larger congruence effect when polarity was present, with a difference of $6.8 \%$ in the green mixed-colour condition versus $3.3 \%$ in the green monochromatic condition. Again, the difference in the monochromatic block could be entirely due to the + pole advantage or reflect a residual context-independent green-positivity link.

Overall, the results show that for red and green colour alike, congruence is significantly larger and, in the current study at least, even only definitely present in colour-polarity-affected (mixed-colour) blocks as compared to colour-polarityunaffected (monochromatic) blocks. Especially when looking at the response pattern for red coloured stimuli, polarity-induced congruence was evidently strong enough to override + pole response facilitation, showing faster and more accurate responses to (red) negative than (red-)positive stimuli, and an inverted pattern, that is, the typical valence asymmetry with + pole response facilitation, when polarity is not present.

\subsection{Congruence Sequence Effects and Valence Modulation}

On the basis of the polarity-correspondence explanation, we expected that, where a colour-valence congruence effect is found, it is asymmetrical: stronger for the + pole than for the - pole stimuli (Lakens, 2012). As explained above, in the current study, this prediction concerned the mixed-colour blocks in which we found that colour-valence congruence effects were indeed asymmetrical. However, in the Introduction, we explained that it is important to consider more conflict control and stronger diminution of congruence effects following incongruent trials for negative targets as an alternative explanation. Therefore, we analysed performance in a preregistered but exploratory analysis from the mixed-colour condition for congruence sequence effects and its valence modulation.

For the factorial analysis, Congruence was coded as described in the previous section (congruent trials: red-negative and green-positive; incongruent trials: red-positive 
and green-negative). ${ }^{8}$ We took into account not only the congruence of the 'current' trial $n$ ( $N$ Congruence: congruent vs. incongruent), but also, if this trial $n$ was preceded by an incongruent trial or not ( $N-1$ Congruence: $n-1$ congruent vs. $n-1$ incongruent). A sequence effect would be reflected in an ANOVA as a significant $N$ Congruence $\times N-1$ Congruence interaction, its modulation would be reflected in a three-way interaction with Valence, with stronger congruence sequence effects (i.e., stronger $N$ Congruence $\times N-1$ Congruence interaction) for negative than for positive word targets. Again, only the data from the condition were analysed, where the mixed block was presented as the first block (Conditions 3 and 4, see Table 1). In all congruence sequence effect analyses, first trials were excluded, since they did not have a value for $N-1$ Congruence. Incorrect trials were excluded in the RT analysis, as well as those trials that followed an incorrect answer, since in such cases it is unclear if the preceding (in)congruence was perceived as such. In Table 5, we provide the means of median correct RTs and mean ERs.

Table 5. Means (SD in Parentheses) of the Median Reaction Times (MRTs, in ms) and the Untransformed Error Rates (MERs, in \%) in the Mixed-Colour Condition per Valence, $N$ Congruence, and N-1 Congruence.

\begin{tabular}{lllll}
\hline Valence & Trial $N-1$ & Trial $N$ & $M_{\mathrm{RT}}(S D)$ & $M_{\mathrm{ER}}(S D)$ \\
\hline Positive & & & & \\
& Congruent & Congruent & $679(81)$ & $2.7(3.9)$ \\
& Incongruent & Congruent & $702(85)$ & $3.6(6.0)$ \\
& Congruent & Incongruent & $737(88)$ & $9.3(7.5)$ \\
& Incongruent & Incongruent & $734(101)$ & $7.0(6.5)$ \\
Negative & & & & \\
& Congruent & Congruent & $706(81)$ & $1.9(2.8)$ \\
& Incongruent & Congruent & $736(97)$ & $4.4(4.7)$ \\
& Congruent & Incongruent & $752(80)$ & $11.9(9.3)$ \\
& Incongruent & Incongruent & $749(120)$ & $7.5(8.2)$ \\
\hline
\end{tabular}

\subsubsection{Reaction Times (RTs)}

A two $($ Valence $) \times$ two $(N$ Congruence $) \times$ two $(N-1$ Congruence $)$ repeatedmeasures ANOVA was performed on the RT data of the mixed condition data. In addition to the main effects for Valence, $F(1,44)=23.13, p<.001, \eta_{\mathrm{p}}^{2}=.345,90 \%$ CI $[.158$, .489], $\eta_{\mathrm{G}}^{2}=.015, \mathrm{BF}_{10}=1261.36$, and $N$ Congruence, $F(1,44)=33.45, p<.001, \eta_{\mathrm{p}}^{2}=$ $.432,90 \%$ CI $[.241, .562], \eta_{\mathrm{G}}^{2}=.039, \mathrm{BF}_{10}=9.80 \times 10^{8}$, which have also been shown 
previously (see mixed-colour condition analysis), there was a marginally significant main effect for $N-1$ Congruence, $F(1,44)=3.39, p=.072, \eta_{\mathrm{p}}^{2}=.072,90 \% \mathrm{CI}[0, .211], \eta_{\mathrm{G}}^{2}=$ $.004, \mathrm{BF}_{10}=1.21$, with faster reactions following $n-1$ congruent than following $n-1$ incongruent trials (see

Table for means). The interaction between $N$ Congruence and Valence was significant, $F(1,44)=4.70, p=.036, \eta_{\mathrm{p}}^{2}=.096,90 \%$ CI $[.003, .243], \eta_{\mathrm{G}}^{2}=.002, \mathrm{BF}_{01}=$ 2.24 , illustrating once more the congruence-effect asymmetry - stronger congruence effects (incongruent minus congruent RTs) for positive (45 ms) than for negative words (29 ms). The interaction between $N$ Congruence and $N-1$ Congruence was also significant, $F(1,44)=8.07, p=.007, \eta_{\mathrm{p}}^{2}=.155,90 \% \mathrm{CI}[.026, .310], \eta_{\mathrm{G}}^{2}=.006, \mathrm{BF}_{10}=$ 7.28 , demonstrating a clear congruence sequence effect - a stronger congruence effect (incongruent minus congruent trials) after $n-1$ congruent trials $(52 \mathrm{~ms})$ than after $n-1$ incongruent trials $(22 \mathrm{~ms})$. The Valence $\times N-1$ Congruence interaction was not significant, $F(1,44)=0.18, p=.670, \eta_{\mathrm{p}}^{2}=.004,90 \% \mathrm{CI}[0, .080], \eta_{\mathrm{G}}^{2}<.001, \mathrm{BF}_{01}=5.95$. Critically, the three-way interaction between $N-1$ Congruence, $N$ Congruence and Valence was not significant, $F(1,44)=0.11, p=.746, \eta_{\mathrm{p}}^{2}=.002,90 \% \mathrm{CI}[0, .068], \eta_{\mathrm{G}}^{2}<$ $.001, \mathrm{BF}_{01}=4.24$. This means that the asymmetry of the congruence effect in the RTs cannot be explained by more conflict regulation with negative targets. RTs for the $n$ congruent and $n$ incongruent trials per Valence and $N-1$ Congruence condition are illustrated in Figure 3. 


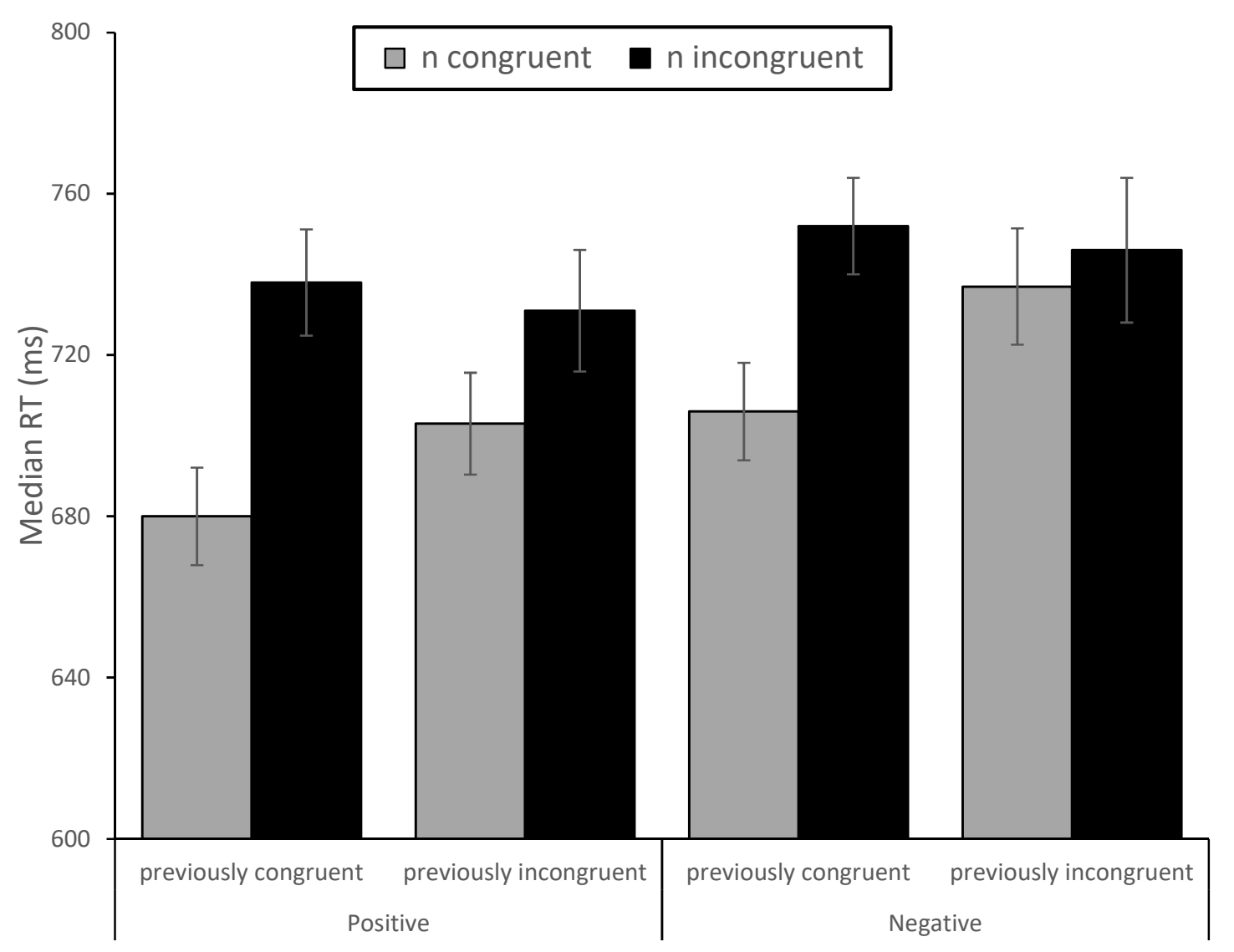

Figure 3. Means of the median correct reaction times (RTs) for positive and negative words from the first mixed-colour block in current $n$ congruent/incongruent and previously $(n-1)$ congruent/incongruent conditions. Error bars show the $S E$ of the means.

\subsubsection{Error Rates (ERs)}

An ANOVA with the same variables was performed for the arcsine transformed error rates. The main effect for $N$ Congruence was significant, $F(1,44)=72.79, p<.001, \eta_{\mathrm{p}}^{2}=$ $.623,90 \%$ CI $[.461, .714], \eta_{\mathrm{G}}^{2}=.177, \mathrm{BF}_{10}=1.47 \times 10^{17}$. Main effects for Valence and $N-1$ Congruence were not significant, $F(1,44)=0.81, p=.373, \eta_{\mathrm{p}}^{2}=.018,90 \%$ CI [0, $.123], \eta_{\mathrm{G}}^{2}=.002, \mathrm{BF}_{01}=4.60$, and $F(1,44)=0.83, p=.368, \eta_{\mathrm{p}}^{2}=.018,90 \% \mathrm{CI}[0, .124]$, $\eta_{\mathrm{G}}^{2}=.001, \mathrm{BF}_{01}=6.50$, respectively. The interaction between $N$ Congruence and Valence was not significant in the ERs, $F(1,44)=0.00, p=.952, \eta_{\mathrm{p}}^{2}<.001,90 \%$ CI $[0,<.001]$, $\eta_{\mathrm{G}}^{2}<.001, \mathrm{BF}_{01}=6.85$, and neither was the interaction between $N-1$ Congruence and Valence, $F(1,44)=0.15, p=.697, \eta_{\mathrm{p}}^{2}=.003,90 \%$ CI $[0, .076], \eta_{\mathrm{G}}^{2}<.001, \mathrm{BF}_{01}=5.97$. 
However, the $N$ Congruence $\times N-1$ Congruence interaction, showing the congruence sequence effect, was significant, $F(1,44)=23.62, p<.001, \eta_{\mathrm{p}}^{2}=.349,90 \%$ CI [.162, .493], $\eta_{\mathrm{G}}^{2}=.042, \mathrm{BF}_{10}=2500.10$. Paired comparisons on the arcsine transformed ERs confirmed a larger congruence effect following preceding congruent $(8.3 \%)$ than preceding incongruent trials $(2.7 \%)$. The $N$ Congruence $\times N-1$ Congruence interaction was also modulated by Valence, $F(1,44)=5.92, p=.019, \eta_{\mathrm{p}}^{2}=.119,90 \% \mathrm{CI}[.011, .270]$, $\eta_{\mathrm{G}}^{2}=.012, \mathrm{BF}_{10}=2.26$. This significant three-way interaction showed that the congruence sequence effect in ERs was stronger for negative words than for positive words. However, this three-way interaction was not responsible for a congruence effect asymmetry in the ERs, as on average the congruence effects for - pole and + pole stimuli were about the same (no $N$ Congruence $\times$ Valence interaction). The three-way interaction in the ERs is illustrated in Figure 4.

Taken together, the findings show clear congruence sequence effects in RTs and ERs. While valence influenced the size of the congruence effect, but not the congruence sequence effect in the RTs, valence did influence the size of congruence sequence effects in the ERs, but since the congruence effects observed in the error rates were quite symmetrical between valence categories (as illustrated in Figure 2, right panel), valencedependent congruence-sequence effects cannot serve as an explanation for congruenceeffect asymmetry. 


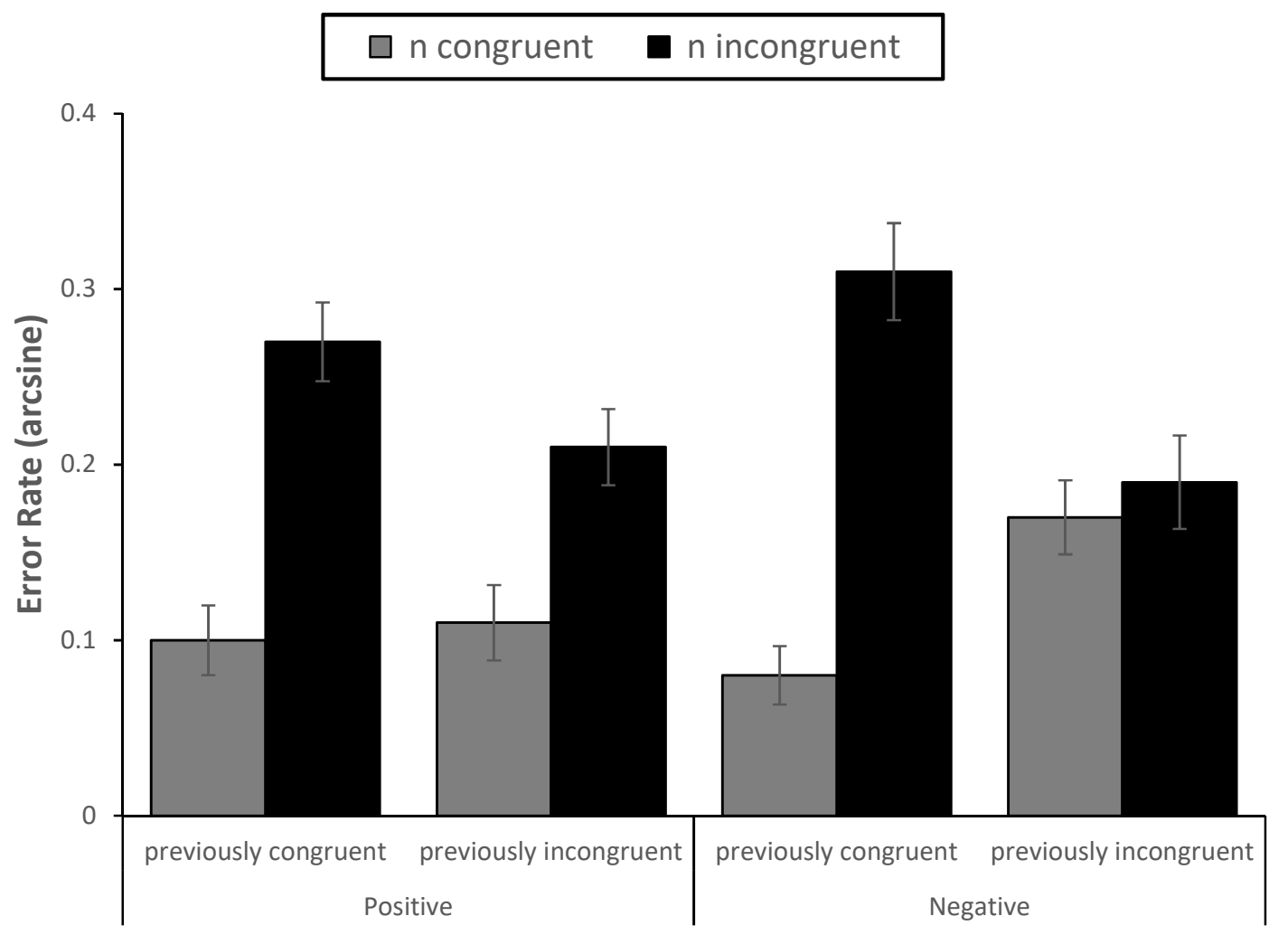

Figure 4. Arcsine transformed error rates for positive and negative words from the first mixed-colour block in current $n$ congruent/incongruent and previously $(n-1)$ congruent/incongruent conditions. The three-way interaction did not lead to an on average just smaller congruence effect in trials following a previously incongruent negative-target trial. Error bars show the $S E$ of the means.

\section{Discussion}

This study investigated the role of polarity, specifically the presence of one versus two colours - red and green - for colour-valence congruence effects in a binary valenceclassification task. Whereas previous studies manipulated the variable colour either within-participant or between-participants, making it hard to paint a unified picture of the nature of influences on colour-valence associations, we methodically manipulated if colours were presented "in opposition" to one another or in isolation: Valence categorisation was performed on positive versus negative words which were either presented in red and green colours intermixed (mixed-colour) or in only one colour, red or green (monochromatic).

Results showed clear differences in congruence effects between polarity-affected and polarity-unaffected trials. To this end, we coded green-positive as well as red- 
negative conditions as congruent and green-negative and red-positive conditions as incongruent and found no clear-cut evidence of a congruence effect in monochromatic blocks where different colour polarities were not present. In these blocks, the remaining advantages for green-positive relative to green-negative and for red-positive relative to red-negative stimuli could have reflected a + pole advantage rather than a colour-valence congruence effect. In contrast, when polarity was present in the mixed-colour blocks, we found congruence effects similar to those reported in other experiments with red-green colour opposition (Kuhbandner \& Pekrun, 2013; Moller et al., 2009; Schietecat et al., 2018b): Responses to congruent trials (red-negative; green-positive) were faster and more accurate than to incongruent trials (red-positive; green-negative). That these differences were indeed due to colour-valence association and not only due to a + pole advantage was reflected in a significant increase of the advantage in green-positive relative to greennegative conditions from monochromatic to mixed-colour blocks and in an advantage of red-negative relative to red-positive conditions in mixed-colour blocks that was missing in the monochromatic blocks. These findings in support of the polarity-correspondence principle are also in line with findings on congruence or compatibility effects in general, where it is often found that the presence of opposite poles of dimensions is necessary for the compatibility effects (cf. Ansorge \& Wühr, 2004; Wühr et al., 2008).

We also corroborated that polarity differences in the valence dimension affect congruence effects (Lakens, 2012; Proctor \& Cho, 2006). Our data showed larger congruence effects for positive than for negative words in the RTs. Such polaritydependent differences have not yet been reported for (chromatic) colour-valence associations. These congruence-effect asymmetries were predicted by + pole response facilitation, a faster processing of + pole than of - pole stimuli (see Lakens, 2012), which we also found in most of our present analyses for the valence targets-with the exception of a polarity-induced red-negative congruence effect, that was pronounced enough to override the advantage for positive words in mixed-colour blocks.

In an additional analysis, we could also rule out that the congruence-effect asymmetry was brought about by a possible conflict control mechanism involving a selectively stronger diminution of congruence effects in negative-target trials, leading to an overall decrease of the congruence effect for negative targets.

Our study shows that red is not linked to negativity in a (monochromatic) speeded binary valence categorisation task, where alternative polarities in the colour dimension are absent. This is in line with an increasing number of studies showing the context- 
dependence of colour-valence congruence effects and demonstrated that polarity correspondence is one factor responsible for how strongly colour-valence associations impact on stimulus processing and overt behaviour. To be precise, we found no evidence that the negativity of red is automatically activated in contexts in which an alternative colour green did not provide an opposite polarity in the colour dimension. From our analysis of the red-monochromatic blocks, it seems that red is not dominantly associated with either positive or negative valence, since evidence for a difference in classification speed of red-positive versus red-negative words was relatively weak. In fact, this finding rather suggests a more neutral if not even slightly positive valence of the colour red without a context of an antagonistic colour pole. Only when green was present alongside red and a bipolar mapping was encouraged, we observed for the colour red a polarity correspondence contribution in the way that responses to negative words were faster than to positive words. All in all, the fact that for red colour, we were able to observe (1) generally relatively small differences between positive and negative valence responses, and (2) an inversion from a (marginal) processing advantage of red-positive (over rednegative) words when polarity is absent to a (marginal) processing advantage of rednegative (over red-positive) words when polarity is present, lend support to the hypothesis that red is ambiguous in nature when it comes to its affective associations. In contrast, for the colour green data might have been more in line with a consistent association between green and positive affect. However, since (1) the difference between green-positive and green-negative responses increased drastically when the counter-colour red was present in the mixed-condition and (2) response patterns did not differ significantly between the monochromatic-red versus the monochromatic-green condition, we cannot rule out that the performance advantage in the monochromatic blocks reflected a general, colourindependent + pole facilitation for the positive words.

What the present study demonstrates is that polarity correspondence is one factor that mediates the connection between the conceptual associations and the overt responses. What the polarity correspondence principle by itself is not able to explain, is, especially in the case of colour, which is not a binary dimension, why polar assignments are made in this way. Apart from the discussion about the potentially ambiguous role of red, the fact that at least a majority of all subjects must have coded green as positive demonstrates that some conceptual knowledge underlying polarity correspondence effects cannot be explained by the polarity correspondence principle itself. However, it is the task of future studies to state more clearly which other factors besides polarity correspondence might 
account for how much weight a conceptual association gains for a behavioural decision (see the dimension-specificity hypothesis by Schietecat et al., 2018a, 2018b, for a recent addition on how context predicts cross-modal associations).

A possible limitation of our study originates from the fact that our colour stimuli were controlled for brightness (luminance), but varied on their average chroma values $\left(C^{*}\right)$, and in effect, in saturation (red: $\mathrm{L}^{*}=51.17, \mathrm{C}^{*}=102, \mathrm{~h}^{*}=40.41$, sat $=1.99$; green: $\mathrm{L}^{*}=51.81, \mathrm{C}^{*}=74.03, \mathrm{~h}^{*}=134.39$, sat $=1.43$, see Table 3$)$. While the influence of brightness on the emotional valence dimension is widely corroborated by various studies and ruled out as a factor in the present study (see Lakens et al., 2012, 2013; Meier et al., 2004; Specker et al., 2018; Specker \& Leder, 2018), variation on other dimensions of colour is an undesirable potential confound. As the study by Wilms and Oberfeld (2018) indicates, highly saturated red might be perceived as neutral, while medium saturated green might be perceived as more positive. Thus, in the case of the mixed blocks, where red was opposed to green, we cannot rule out that the responses to the emotional word stimuli were affected by different saturations for the red versus the green stimuli. However, some studies suggest that saturation has a stronger behavioural and/or physiological effect on the arousal or activity dimension of emotions, less so on the valence dimension (Schietecat et al., 2018a; Valdez \& Mehrabian, 1994; Zieliński, 2016). Still, in the case of the mixed blocks, even if the saturation difference between our red and green is with approximately 0.56 relatively small, we cannot rule out that the response times to the emotional word stimuli were affected by different saturations for the red versus the green stimuli.

Another limitation is that it is not easy to study the generality of the polarity correspondence principle in the realm of colour-emotion associations. The reason for this is that colour can be a difficult research subject if we want to exert proper control over the responsible factors by which colours differ from one another. For instance, while it would be interesting to test green against less ambiguously associated colours than red, the questions as to what colour combination could be tested is not easy to answer: Firstly, colours may have all sorts of associations, but positive feelings seem to be predominant, which, on a related note, is reflected in valence judgements for colours. As mentioned, in Wilms and Oberfeld (2018), some colours receive higher valence ratings than others, but no colour is judged as negative per se. Secondly, while achromatic colours are often described as less positive than chromatic ones (Adams \& Osgood, 1973; Kaya \& Epps, 2004), equating the colourimetrics is impossible in this case. 


\section{Conclusion}

Our study is the first in colour-valence-association research to directly oppose polarityaffected to polarity-unaffected trials. Our results underscore once more the green-positive and red-negative associations, but demonstrate one important influence on whether or not the associations modulate behaviour: the presence of polarities on both valence and colour dimensions. The structural overlap in the mapping of colour and valence concepts on + and - poles is, thus, one factor for if or if not these associations really play out. Our study is also the first to rule out an alternative origin of the asymmetry of the polaritycorrespondence effect in terms of more conflict regulation with - pole than with + pole stimuli.

\section{Open Practices Statement}

The study was preregistered via https://osf.io/c4zry (Foster \& Deardorff, 2017). The source code for the experiment is available via the same link, as well as all behavioural data collected.

\section{Acknowledgments}

Claudia Kawai and Gáspár Lukács are recipients of a DOC Fellowship of the Austrian Academy of Sciences at the Department of Cognition, Emotion, and Methods in Psychology at the University of Vienna. The research was also supported by Dr. Franziska Pinsker. 


\section{References}

Adams, F. M., \& Osgood, C. E. (1973). A cross-cultural study of the affective meanings of color. Journal of Cross-Cultural Psychology, 4(2), 135-156. doi: $10.1177 / 002202217300400201$

Akers, A., Barton, J., Cossey, R., Gainsford, P., Griffin, M., \& Micklewright, D. (2012). Visual color perception in green exercise: Positive effects on mood and perceived exertion. Environmental Science \& Technology, 46(16), 8661-8666. doi: $10.1021 / \mathrm{es} 301685 \mathrm{~g}$

Ansorge, U., Kunde, W., \& Kiefer, M. (2014). Unconscious vision and executive control: How unconscious processing and conscious action control interact. Consciousness and Cognition, 27, 268-287. doi: 10.1016/j.concog.2014.05.009

Ansorge, U., \& Wühr, P. (2004). A response-discrimination account of the Simon effect. Journal of Experimental Psychology: Human Perception and Performance, 30(2), 365-377. doi: 10.1037/0096-1523.30.2.365

Bertelson, P. (1965). Serial choice reaction-time as a function of response versus signaland-response repetition. Nature, 206(4980), 217. doi: 10.1038/206217a0

Botvinick, M. M., Braver, T. S., Barch, D. M., Carter, C. S., \& Cohen, J. D. (2001). Conflict monitoring and cognitive control. Psychological Review, 108(3), 624652. doi: 10.1037/0033-295X.108.3.624

Briki, W., \& Hue, O. (2016). How red, blue, and green are affectively judged: Affective judgments of colors. Applied Cognitive Psychology, 30(2), 301-304. doi: 10.1002/acp.3206

Briki, W., Rinaldi, K., Riera, F., Trong, T. T., \& Hue, O. (2015). Perceiving red decreases motor performance over time: A pilot study. European Review of Applied Psychology, 65(6), 301-305. doi: 10.1016/j.erap.2015.09.001

Brysbaert, M., Buchmeier, M., Conrad, M., Jacobs, A. M., Bölte, J., \& Böhl, A. (2011). The word frequency effect: A review of recent developments and implications for the choice of frequency estimates in German. Experimental Psychology, 58(5), 412-424. doi: 10.1027/1618-3169/a000123

Buechner, V. L., \& Maier, M. A. (2016). Not always a matter of context: Direct effects of red on arousal but context-dependent moderations on valence. PeerJ, 4, e2515. doi: $10.7717 /$ peerj. 2515 
Clark, H. H. (1969). Linguistic processes in deductive reasoning. Psychological Review, 76(4), 387-404. doi: 10.1037/h0027578

Clarke, T., \& Costall, A. (2008). The emotional connotations of color: A qualitative investigation. Color Research \& Application, 33(5), 406-410. doi: $10.1002 / \mathrm{col} .20435$

Crozier, W. R. (1999). The meanings of colour: Preferences among hues. Pigment \& Resin Technology, 28(1), 6-14. doi: 10.1108/03699429910252315

De Bruijn, E. R., Hulstijn, W., Verkes, R. J., Ruigt, G. S., \& Sabbe, B. G. (2004). Druginduced stimulation and suppression of action monitoring in healthy volunteers. Psychopharmacology, 177(1-2), 151-160. doi:10.1007/s00213-0041915-6

Delacre, M., Lakens, D., \& Leys, C. (2017). Why psychologists should by default use Welch's t-test instead of Student's t-test. International Review of Social Psychology, 30(1), 92. doi: 10.5334/irsp.82

Dreisbach, G., \& Fischer, R. (2012). Conflicts as aversive signals. Brain and Cognition, 78(2), 94-98. doi:10.1016/j.bandc.2011.12.003

Elliot, A. J., \& Maier, M. A. (2012). Color-in-context theory. In Advances in Experimental Social Psychology, 45, 61-125. doi: 10.1016/B978-0-12-3942869.00002-0

Elliot, A. J., Maier, M. A., Moller, A. C., Friedman, R., \& Meinhardt, J. (2007). Color and psychological functioning: The effect of red on performance attainment. Journal of Experimental Psychology. General, 136(1), 154-168. doi: 10.1037/0096-3445.136.1.154

Elliot, A. J., \& Niesta, D. (2008). Romantic red: Red enhances men's attraction to women. Journal of Personality and Social Psychology, 95(5), 1150-1164. doi: 10.1037/0022-3514.95.5.1150

Elliot, A. J., Niesta Kayser, D., Greitemeyer, T., Lichtenfeld, S., Gramzow, R. H., Maier, M. A., \& Liu, H. (2010). Red, rank, and romance in women viewing men. Journal of Experimental Psychology: General, 139(3), 399-417. doi: 10.1037/a0019689

Faul, F., Erdfelder, E., Buchner, A., \& Lang, A.-G. (2009). Statistical power analyses using $\mathrm{G}^{*}$ Power 3.1: Tests for correlation and regression analyses. Behavior Research Methods, 41, 1149-1160. 
Fetterman, A. K., Robinson, M. D., \& Meier, B. P. (2012). Anger as "seeing red": Evidence for a perceptual association. Cognition \& Emotion, 26(8), 1445-1458. doi: 10.1080/02699931.2012.673477

Foster, E. D., \& Deardorff, A. (2017). Open Science Framework (OSF). Journal of the Medical Library Association, 105(2), 203. doi: 10.5195/jmla.2017.88

Francis, G. (2013). Publication bias in "Red, rank, and romance in women viewing men," by Elliot et al. (2010). Journal of Experimental Psychology: General, 142(1), 292296. doi: $10.1037 / \mathrm{a} 0027923$

Fritz, J., \& Dreisbach, G. (2013). Conflicts as aversive signals: Conflict priming increases negative judgments for neutral stimuli. Cognitive, Affective, \& Behavioral Neuroscience, 13(2), 311-317. doi:10.3758/s13415-012-0147-1

Goller, F., Kroiss, A., \& Ansorge, U. (2019). Conflict-elicited negative evaluations of neutral stimuli: Testing overt responses and stimulus-frequency differences as critical side conditions. Frontiers in Psychology, 10, 2204. doi:10.3389/fpsyg.2019.02204

Gratton, G., Coles, M. G., \& Donchin, E. (1992). Optimizing the use of information: Strategic control of activation of responses. Journal of Experimental Psychology: General, 121(4), 480.

Greenwald, A. G., McGhee, D. E., \& Schwartz, J. L. (1998). Measuring individual differences in implicit cognition: The implicit association test. Journal of Personality and Social Psychology, 74(6), 1464-1480. http://dx.doi.org/10.1037/0022-3514.74.6.1464

Hobson, N. M., Saunders, B., Al-Khindi, T., \& Inzlicht, M. (2014). Emotion downregulation diminishes cognitive control: A neurophysiological investigation. Emotion, 14(6), 1014-1026. doi:10.1037/a0038028

Huber-Huber, C., \& Ansorge, U. (2017). The role of RT carry-over for congruence sequence effects in masked priming. Journal of Experimental Psychology: Learning, Memory, and Cognition, 43(5), 757-780. doi: 10.1037/xlm0000331

Inzlicht, M., Bartholow, B. D., \& Hirsh, J. B. (2015). Emotional foundations of cognitive control. Trends in Cognitive $\quad$ Sciences, 19(3), 126-132. doi:10.1016/j.tics.2015.01.004

Jonauskaite, D., Wicker, J., Mohr, C., Dael, N., Havelka, J., Papadatou-Pastou, M., Zhang, Oberfeld, D. (2019). A machine learning approach to quantify the 
specificity of colour-emotion associations and their cultural differences. Royal Society Open Science, 6(9), 190741. doi: 10.1098/rsos.190741

Kaya, N., \& Epps, H. H. (2004). Relationship between color and emotion: A study of college students. College Student Journal, 38(3), 396-405.

Kelley, K. (2019). MBESS: The MBESS R Package. R package version 4.5.1. Retrieved from https://CRAN.R-project.org/package=MBESS

Kuhbandner, C., \& Pekrun, R. (2013). Joint effects of emotion and color on memory. Emotion, 13(3), 375-379. doi: 10.1037/a0031821

Labrecque, L. I., \& Milne, G. R. (2012). Exciting red and competent blue: The importance of color in marketing. Journal of the Academy of Marketing Science, 40(5), 711727. doi: 10.1007/s11747-010-0245-y

Lakens, D. (2012). Polarity correspondence in metaphor congruency effects: Structural overlap predicts categorization times for bipolar concepts presented in vertical space. Journal of Experimental Psychology: Learning, Memory, and Cognition, 38(3), 726-736. doi: 10.1037/a0024955

Lakens, D. (2013). Calculating and reporting effect sizes to facilitate cumulative science: A practical primer for t-tests and ANOVAs. Frontiers in Psychology, 4, 863. doi: 10.3389/fpsyg.2013.00863

Lakens, D., Fockenberg, D. A., Lemmens, K. P. H., Ham, J., \& Midden, C. J. H. (2013). Brightness differences influence the evaluation of affective pictures. Cognition \& Emotion, 27(7), 1225-1246. doi: 10.1080/02699931.2013.781501

Lakens, D., Semin, G. R., \& Foroni, F. (2012). But for the bad, there would not be good: Grounding valence in brightness through shared relational structures. Journal of Experimental Psychology: General, 141(3), 584-594. doi: 10.1037/a0026468

Lawrence, M. A. (2016). Ez: Easy analysis and visualization of factorial experiments. R package version 4.4-0. Retrieved from https://CRAN.R-project.org/package=ez

Lebois, L. A., Wilson-Mendenhall, C. D., \& Barsalou, L. W. (2015). Are automatic conceptual cores the gold standard of semantic processing? The contextdependence of spatial meaning in grounded congruency effects. Cognitive Science, 39(8), 1764-1801. doi: 10.1111/cogs.12174

Lehmann, G. K., \& Calin-Jageman, R. J. (2017). Is red really romantic?: Two preregistered replications of the red-romance hypothesis. Social Psychology, 48(3), 174-183. doi: 10.1027/1864-9335/a000296 
Lehmann, G. K., Elliot, A. J., \& Calin-Jageman, R. J. (2018). Meta-analysis of the effect of red on perceived attractiveness. Evolutionary Psychology, 16(4), 147470491880241. doi: 10.1177/1474704918802412

Lichtenfeld, S., Elliot, A. J., Maier, M. A., \& Pekrun, R. (2012). Fertile green: Green facilitates creative performance. Personality and Social Psychology Bulletin, 38(6), 784-797. doi: 10.1177/0146167212436611

Lichtenfeld, S., Maier, M. A., Elliot, A. J., \& Pekrun, R. (2009). The semantic red effect: Processing the word red undermines intellectual performance. Journal of Experimental Social Psychology, 45(6), 1273-1276. doi: 10.1016/j.jesp.2009.06.003

Lohmann, C., \& Jorschick, A. (2015, March). Studie in Rot-Grün - Farben aktivieren emotionale Wortbedeutungen [Study in red-green - colors activate emotional word meanings]. Abstract presented at the TEAP 2015 Conference, Hildesheim, Germany.

Lukács, G. (2019). neatStats: An R Package for neat and painless statistical reporting. R package version 0.3.1. Retrieved from https:/github.com/gasparl/neatstats

Makowski, D., Ben-Shachar, M., \& Lüdecke, D. (2019). bayestestR: Describing effects and their uncertainty, existence and significance within the Bayesian framework. Journal of Open Source Software, 4(40), 1541. doi: 10.21105/joss.01541

Mathôt, S. (2017, May 15). Bayes like a Baws: Interpreting Bayesian Repeated Measures in JASP. https://www.cogsci.nl/blog/interpreting-bayesian-repeated-measuresin-jasp

Mehta, R., \& Zhu, R. (2009, February). Blue or red? Exploring the effect of color on cognitive task performances. Science, 323(5918), 1226-1229. doi: 10.1126/science.1169144

Meier, B. P., \& Robinson, M. D. (2004). Why the sunny side is up: Associations between affect and vertical position. Psychological Science, 15(4), 243-247. doi: 10.1111/j.0956-7976.2004.00659.x

Meier, B. P., Robinson, M. D., \& Clore, G. L. (2004). Why good guys wear white: Automatic inferences about stimulus valence based on brightness. Psychological Science, 15(2), 82-87. doi: 10.1111/j.0963-7214.2004.01502002.x

Moller, A. C., Elliot, A. J., \& Maier, M. A. (2009). Basic hue-meaning associations. Emotion, 9(6), 898-902. doi: 10.1037/a0017811 
Moors, A., \& De Houwer, J. (2006). Automaticity: A theoretical and conceptual analysis. Psychological

Bulletin, 132(2),

297-326. http://dx.doi.org/10.1037/0033-2909.132.2.297

Morey, R. D., \& Rouder, J. N. (2018). BayesFactor: Computation of Bayes factors for common designs. R package version 0.9.12-4.2. Retrieved from https://CRAN.Rproject.org/package=BayesFactor

Pazda, A. D., Elliot, A. J., \& Greitemeyer, T. (2012). Sexy red: Perceived sexual receptivity mediates the red-attraction relation in men viewing woman. Journal of Experimental Social Psychology, 48(3), 787-790. doi: 10.1016/j.jesp.2011.12.009

Peirce, J. W. (2007). PsychoPy-Psychophysics software in Python. Journal of Neuroscience Methods, 162(1), 8-13. doi: 10.1016/j.jneumeth.2006.11.017

Peperkoorn, L. S., Roberts, S. C., \& Pollet, T. V. (2016). Revisiting the Red Effect on attractiveness and sexual receptivity: No effect of the color red on human mate preferences. Evolutionary Psychology, 14(4), 147470491667384. doi: $10.1177 / 1474704916673841$

Pravossoudovitch, K., Cury, F., Young, S. G., \& Elliot, A. J. (2014). Is red the colour of danger? Testing an implicit red-danger association. Ergonomics, 57(4), 503-510. doi: 10.1080/00140139.2014.889220

Proctor, R. W., \& Cho, Y. S. (2006). Polarity correspondence: A general principle for performance of speeded binary classification tasks. Psychological Bulletin, 132(3), 416-442. doi: 10.1037/0033-2909.132.3.416

Puccioni, O., \& Vallesi, A. (2012). Sequential congruency effects: Disentangling priming and conflict adaptation. Psychological Research, 76(5), 591-600. doi: $10.1007 / \mathrm{s} 00426-011-0360-5$

R Core Team. (2019). R: A language and environment for statistical computing. R Foundation for Statistical Computing, Vienna, Austria. Retrieved from https://www.R-project.org/

Rogers, R. D., \& Monsell, S. (1995). Costs of a predictable switch between simple cognitive tasks. Journal of Experimental Psychology: General, 124(2), 207-231. http://doi.org/10.1037/0096-3445.124.2.207

Saito, M. (1996). Comparative studies on color preference in Japan and other Asian regions, with special emphasis on the preference for white. Color Research and 
Application, 21(1), 35-49. doi: 10.1002/(SICI)15206378(199602)21:1<35::AID-COL4>3.0.CO;2-6

Schietecat, A. C., Lakens, D., IJsselsteijn, W. A., \& De Kort, Y. A. W. (2018a). Predicting context-dependent cross-modal associations with dimension-specific polarity attributions. Part 1 - Brightness and aggression. Collabra: Psychology, 4(1), 14. doi: $10.1525 /$ collabra. 110

Schietecat, A. C., Lakens, D., IJsselsteijn, W. A., \& De Kort, Y. A. W. (2018b). Predicting context-dependent cross-modal associations with dimension-specific polarity attributions. Part 2: Red and valence. Collabra: Psychology, 4(1), 21. doi: 10.1525/collabra.126

Schloss, K. B., Strauss, E. D., \& Palmer, S. E. (2013). Object color preferences. Color Research and Application, 38(6), 393-411. doi: 10.1002/col.21756

Schouppe, N., Braem, S., De Houwer, J., Silvetti, M., Verguts, T., Ridderinkhof, K. R., \& Notebaert, W. (2015). No pain, no gain: The affective valence of congruency conditions changes following a successful response. Cognitive, Affective, \& Behavioral Neuroscience, 15(1), 251-261. doi: 10.3758/s13415-014-0318-3

Smajic, A., Merritt, S., Banister, C., \& Blinebry, A. (2014). The red effect, anxiety, and exam performance: A multistudy examination. Teaching of Psychology, 41(1), 37-43. doi: 10.1177/0098628313514176

Sokal, R. R., \& Rohlf, R. F. (1981). Biometry: The principles and practice of statistics in biological research ( $2^{\text {nd }}$ ed.). San Francisco, CA: Freeman.

Specker, E., \& Leder, H. (2018). Looking on the bright side: Replicating the association between brightness and positivity. Collabra: Psychology, 4(1), 34. doi: 10.1525/collabra.168

Specker, E., Leder, H., Rosenberg, R., Hegelmaier, L. M., Brinkmann, H., Mikuni, J., \& Kawabata, H. (2018). The universal and automatic association between brightness and positivity. Acta Psychologica, 186, 47-53. doi: 10.1016/j.actpsy.2018.04.007

Steele, K. M. (2014). Failure to replicate the Mehta and Zhu (2009) color-priming effect on anagram solution times. Psychonomic Bulletin \& Review, 21(3), 771-776. doi: $10.3758 / \mathrm{s} 13423-013-0548-3$

Steiger, J. H. (2004). Beyond the F test: Effect size confidence intervals and tests of close fit in the analysis of variance and contrast analysis. Psychological Methods, 9(2), 164-182. doi: 10.1037/1082-989X.9.2.164 
Terwogt, M. M., \& Hoeksma, J. B. (1995). Colors and emotions: Preferences and combinations. The Journal of General Psychology, 122(1), 5-17. doi: 10.1080/00221309.1995.9921217

van Steenbergen, H., Band, G. P., \& Hommel, B. (2009). Reward counteracts conflict adaptation: Evidence for a role of affect in executive control. Psychological Science, 20(12), 1473-1477. doi: 10.1111/j.1467-9280.2009.02470.x

van Steenbergen, H., Band, G. P., \& Hommel, B. (2010). In the mood for adaptation: How affect regulates conflict-driven control. Psychological Science, 21(11), 1629-1634. doi: 10.1177/0956797610385951

Võ, M., Conrad, M., Kuchinke, L., Urton, K., Hofmann, M., \& Jacobs, A. (2009). The Berlin Affective Word List Reloaded (BAWL-R). Behavior Research Methods, 41(2), 534-538. doi: 10.3758/BRM.41.2.534

Wilms, L., \& Oberfeld, D. (2018). Color and emotion: Effects of hue, saturation, and brightness. Psychological Research, 82(5), 896-914. doi: 10.1007/s00426-0170880-8

Wilson, G. D. (1966). Arousal properties of red versus green. Perceptual and Motor Skills, 23(3), 947-949. doi: 10.2466/pms.1966.23.3.947

Wolfe, J. M., Butcher, S. J., Lee, C., \& Hyle, M. (2003). Changing your mind: On the contributions of top-down and bottom-up guidance in visual search for feature singletons. Journal of Experimental Psychology: Human Perception and Performance, 29(2), 483 -502. doi: 10.1037/0096-1523.29.2.483

Wu, Y., Lu, J., van Dijk, E., Li, H., \& Schnall, S. (2018). The color red is implicitly associated with social status in the United Kingdom and China. Frontiers in Psychology, 9, 1902. doi: 10.3389/fpsyg.2018.01902

Wühr, P., Biebl, R., \& Ansorge, U. (2008). The impact of stimulus and response variability on SR correspondence effects. Journal of Experimental Psychology: Learning, Memory, and Cognition, 34(3), 533-545. doi: 10.1037/02787393.34.3.5 


\section{Endnotes}

${ }^{1}$ Emotions are states of the human mind that are typically characterized by a particular subjective feeling (e.g., anger, sadness, joy), accompanied by typical cognitive representations (e.g., beliefs about the state of affairs represented by the emotion; e.g., that somebody took my sun cream away from me on purpose), physiological activity (in the brain and in the peripheral nervous system; e.g., activation of the pituitary gland), and by action tendencies (e.g., aggressive behavior in the case of anger). In contrast, the term valence only fetches that part of the feeling of an emotion having to do with its felt overall positivity versus negativity.

${ }^{2}$ For most of the current manuscript, we use the term concept to cover both, more abstract semantic representations and more concrete sensory or sensorimotor representations. Only occasionally, we refer to the latter as features. However, for our argument about the contribution of polarity correspondence to colour-valence congruence effects, the distinction between abstract semantic concepts and concrete sensory features is peripheral. Therefore, for the sake of convenience, on most occasions we jointly refer to both, colours and emotions, by the single term concept.

3 Some studies that tested not exactly colour-emotion congruence effects themselves but related effects, such as influences of specific colour on attractiveness ratings of faces of varying attractiveness rather than of different valence stimuli, used rating tasks and allowed even intermediate or neutral judgments (e.g., Elliott \& Niesta, 2008). Though in these tasks, the mere presence of stimuli on opposite poles of dimensions (e.g., of + and - pole stimuli on an attractiveness dimension) might have been critical for the found colour-based effects, this question is beyond the scope of the present study and not central to our major tests.

${ }^{4}$ In the preregistration, we stated that we opened 180 slots for participation (i.e., giving a maximum of 180 people the possibility to take part in our experiment). Beforehand, it was not possible to say how many participants would sign up in the university's participant-registration system, but typically, at least around two thirds of all slots are filled up. The smallest targeted sample size (see Methods) was approximately 40 participants per group. Testing took place within a span of 3 days. Out of the 180 open spaces during these 3 testing days, a total of 145 people could be tested in actuality.

${ }^{5}$ Note that the number of positively rated (e.g., 43) and negatively rated words (e.g., 52) does not necessarily have to add up to 120 . In the example, 43 out of 60 positive words were "correctly" judged by the participant as, in fact, positive, while 17 of the 60 
positive words were judged as negative. Furthermore, the participant judged 52 out of the 60 negative words as, in fact, negative, but judged eight of the negative words as positive (e.g., 'naïve' is a negative term according to the BAWL-R database, but could be judged as a more positive concept by some participants).

${ }^{6}$ For the + pole response facilitation, it is not necessary that both dimensions contributing to a polarity correspondence effect are realized within the same context. As long as there are polar opposites regarding one dimension, here of valence, + pole facilitation for this dimension can be observed.

${ }^{7}$ This ANOVA for the mixed blocks was not preregistered. Originally, we preregistered a three-way mixed model ANOVA on the conjoined first block data (from the mixed and monochromatic conditions), the variables being Valence (within participant), Colour-Valence Congruence (within) and Colour Condition (monochromatic vs. mixed, between) if the first ANOVA for the monochromatic blocks shows a Colour $\times$ Valence interaction (i.e., a congruence effect). The reasoning was that the effects for the mixed blocks are discernible from the two preregistered analyses of only the monochromatic condition and mono- and mixed-coloured condition together. However, this analysis proved to be not feasible, as it relies partly on within-participant and partly on between-participants data from the same participants. For this reason, we opted for the two separate analyses.

${ }^{8}$ The preregistered analysis for congruence sequence effects in the mixed-colour condition comprised the factors $N$ Congruence, $N-1$ Congruence, Valence and, additionally, Colour. This was a logical fallacy, since Congruence itself already describes a specific directional relationship between Colour and Valence. Thus, in the scope of this analysis, Colour was not a necessary variable and therefore not included. 


\section{Appendix}

Positive Words

SPANNEND

TRIUMPH

SCHLAU

ERINNERN

FEIERN

VERTRAUT

WÜNSCHEN

HELD

BELOHNEN

AKTIV

PARTY

TOLERANT

TAPFER

BEGEISTERN

FREIZEIT

REIZVOLL

RETTEN

ANMUTIG

ENTDECKEN

MUTIG

LEUCHTEN

MÖGEN

FREUEN

GENIE

FLIRT

SCHÜTZEN

TALENT

EKSTASE

BUNT

STARK

GRANDIOS

SPENDE

BEQUEM

SCHENKEN

VERBESSERN

BEIFALL

LEBENDIG

ZUSTIMMEN

UMARMEN

INTENSIV

ANGEBOT

ERFINDER

ANSPORN

MAGISCH

OPTIMIST

STAUNEN

APPETIT

HEITER

REICHTUM

SANFT

GRINSEN

STÄRKEN

LUXUS

MUNTER

DUFTEN

HEIMKEHR

GEBURTSTAG

GRATIS

LOB

BEGRÜSSEN

Negative Words

NAIV

AAS

UNBEQUEM

BEREUEN

SPUCKEN

VERBIETEN

MIETE

SCHMUTZ

UNFAIR

BALLAST

PEINLICH

SCHWACH

MAUL

ZWINGEN

FORDERUNG

TRETEN

HEUCHELN

PRIMITIV

STOTTERN

TRAUERN

DROHEN

ALLEIN

WARTEREI

GEFÄNGNIS

VERZICHT

ABSTIEG

PANISCH

MONOTON

REGLOS

LEIDEN

MÜSSEN

STRAFE

TROSTLOS

FEHLEN

GRAUSAM

VERLETZEN

KÜNDIGEN

SCHEUSAL

BOSHAFT

ZÖGERN

IDIOT

BLAMAGE

BITTER

MANGEL

VERBRECHEN

NARBIG

KORRUPT

HEULEN

SCHLAPP

ARROGANZ 
SCHLIESSEN

HÄNGEN

$\mathrm{LOCH}$
GEMEIN

GIERIG

STÖREN

TROTZIG

VERSAGEN 